\title{
23. GRAIN-SIZE DISTRIBUTION OF SEDIMENTS FROM THE EASTERN INDIAN OCEAN: DEEP SEA DRILLING PROJECT, LEG 27
}

\author{
Paul A. Thayer, John Hostettler, and Scott Smith, \\ University of North Carolina at Wilmington, Wilmington, North Carolina
}

\begin{abstract}
Grain size of 172 unconsolidated sediment samples from five DSDP sites in the eastern Indian Ocean was determined by sieve and pipette techniques. Folk and Ward (1957) statistical parameters were calculated for all samples by computer.

On the basis of grain-size parameters, the sediments can be divided into two broad groups: Mesozoic clays and Cenozoic calcareous oozes. Mesozoic sediments have a mean size of $10.07 \phi$ and are very poorly sorted $(2.58 \phi)$, coarse skewed $(-0.14)$, and mesokurtic $\left(K_{G}^{\prime}{ }_{G}=\right.$ 0.47 ). Cenozoic sediments have a mean size of $7.67 \phi$ and are very poorly sorted $(3.19 \phi)$, fine skewed $(+0.18)$, and mesokurtic $\left(K_{G}^{\prime}=\right.$ 0.47 ).
\end{abstract}

\section{INTRODUCTION}

Although grain size is one of the fundamental properties of deep-sea sediments and sedimentary rocks (Griffiths, 1967; Blatt et al., 1972), little detailed work on grain-size distribution or statistical parameters has previously been done on samples obtained by the Deep Sea Drilling Project. Sand-silt-clay percentages are routinely determined for all Initial Reports of the Deep Sea Drilling Project, but only Lisitzin et al. (1971) for Leg 6 and Boyce (1972) for Leg 11 have performed complete grain-size analyses at $1 \phi$ unit intervals. However, neither investigator computed statistical parameters of grain-size distribution. Beall et al. (1973) did detailed sieve analyses on the sand and coarse silt fractions for Leg 10 sediments, but did not consider the rest of the silt or clay fractions.

By comparison, grain-size data for different types of Recent deep-sea sediments have been reported by a number of workers (e.g., Barth et al., 1939; Sverdrup et al., 1942; Kuenen and Neeb, 1943; Revelle, 1944; Shukri and Higazy, 1944; Kuenen, 1950; Correns, 1955; van Andel, 1964; van Andel and Veevers, 1967; Kukal, 1971; and Lisitzin, 1972). Unfortunately, most of these data are presented in the form of histograms and usually no statistical parameters are computed; or if they are, the outmoded quartile measures of Trask (1932) have been generally used. Thus, much of the grain-size data from these studies cannot be compared with modern statistical data obtained from ancient deep-sea sediments except in a very general sense. Furthermore, data from the modern sediments are of little value for trying to recognize environments and conditions of deposition in older deep-sea deposits.

This paper presents detailed grain-size information on Mesozoic and Cenozoic deep-sea sediments cored at five sites in the eastern Indian Ocean (Figure 1). Our chief objective is to provide basic size-distribution data for different lithologic units cored at these sites. These data, along with petrologic data, are utilized in Chapter 48 (this volume), for deducing environments of deposition and physical mechanisms of sedimentation. The information may alşo be useful for relating size distribution to physical properties of the sediments, such as porosity, bulk density, sonic transmissability, tensile strength, etc.

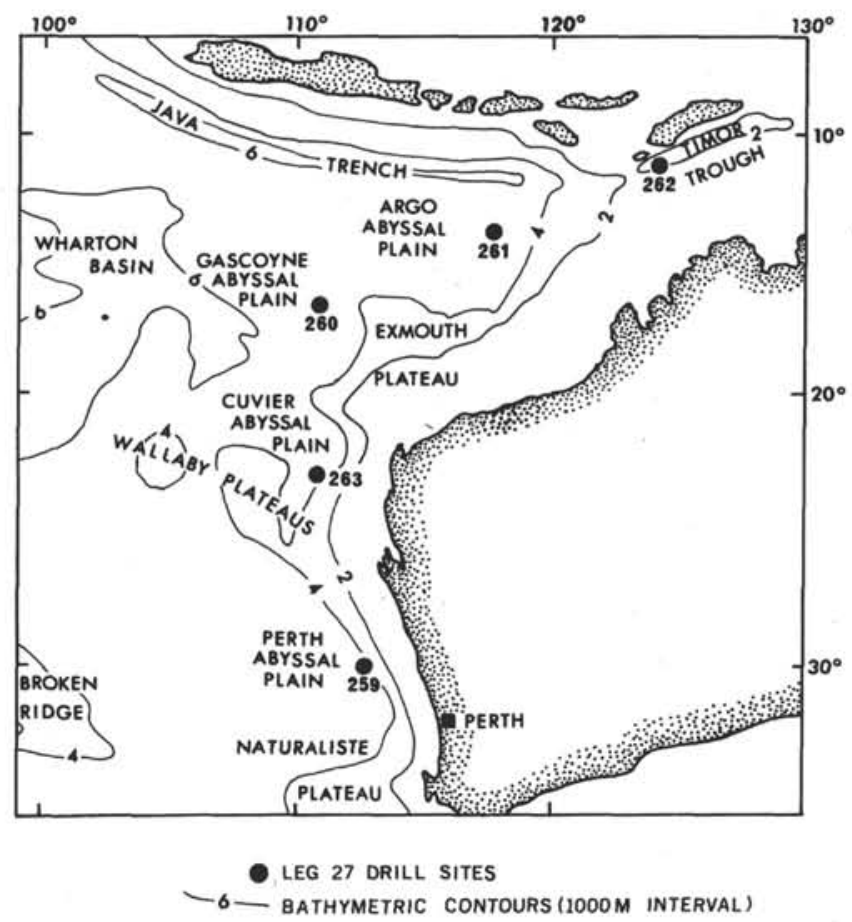

Figure 1.Location map showing Leg 27 drill sites and general physiographic features of the eastern Indian Ocean. 


\section{METHODS}

Unconsolidated sediment samples (172) were selected for grain-size analysis. Sample locations are shown in Figure 2. Size analyses on lithified or semilithified sediments were not performed because the rigorous treatment necessary for disaggregation leads to an erroneous picture of the grain-size distribution (Folk, 1968; Gealy, 1971). Thus, size data presented here are biased in favor of unconsolidated sedimentary units.

Water-soluble salts were removed from all samples by dialysis (Müller, 1967). Each sample was placed in a dialyzer bag and put in a large container of circulating distilled water for 2 days. Samples were then dispersed by soaking for $24 \mathrm{hr}$ in $200 \mathrm{ml}$ of distilled water to which $50 \mathrm{ml}$ of $10 \%$ sodium hexametaphosphate (calgon) was added. If lumps of mud were still present after soaking, they were removed by gentle crushing with a rubbergloved finger (Folk, 1968). The sediment was wet sieved on a $62.5 \mu$ screen to separate the sand and mud (silt plus clay) fractions. The sand fraction was dried and sieved on optically calibrated screens at $1 / 2 \phi$ unit intervals for 15 min on a Ro-tap machine (Ingram, 1971). Grain size of the mud fraction was determined in a constant temperature room by pipette technique (Folk, 1968; Galehouse, 1971), which is based on settling velocities of particles calculated from Stokes' Law (Krumbein and Pettijohn, 1938). Pipette withdrawals were taken at times corresponding to $1 / 2$ unit intervals from $4.5 \phi$ to $6 \phi$ and at $1 \phi$ unit intervals from $7 \phi$ to $11 \phi$. The pipette analyses were terminated at $11 \phi(0.49 \mu)$ because particles smaller than this diameter are strongly affected by Brownian movement of the water in which they are suspended (Irani and Callis, 1963).

Cumulative percentages of sand and mud were determined by computer and cumulative curves drawn on arithmetic graph paper. If the cumulative percentage at $11 \phi$ was less than $95 \%$, the unsampled fine population was interpolated by extending the cumulative curve in a straight line from $11 \phi$ to $14 \phi$ at $100 \%$ (Folk, 1968). This operation assumes all sediment is coarser than $14 \phi$ and that the clay mode lies near $12 \phi$. Cumulative percentage values for $12 \phi$ and $13 \phi$ were read directly from the interpolated curve.

Folk and Ward (1957), Inman (1962), and momentmeasure (Griffiths, 1967) statistics were calculated by computer. Folk and Ward statistics are used in this paper since they now are most widely used by sedimentologists. Inman and moment-measure statistics calculated for these samples can be obtained by writing the senior author.

The Folk and Ward (1957) measure for average sediment size is the graphic mean $\left(M_{z}\right)$, given by the formula

$$
M_{z}=\frac{\phi 16+\phi 50+\phi 84}{3}
$$

Their measure for determining the uniformity of grain size (sorting) of sediments is called inclusive graphic standard deviation and is found by the formula

$$
\sigma_{I}=\frac{\phi 84-\phi 16}{4}+\frac{\phi 95-\phi 5}{6.6}
$$

Plotting of hundreds of analyses from many different environments led Folk and Ward (1957) to suggest the following verbal classification for sorting: $\sigma_{I}$ under $0.35 \phi$, very well sorted; ${ }^{\sigma} I 0.35$ to $0.50 \phi$, well sorted; ${ }^{\sigma} I$ 0.50 to $1.00 \phi$, moderately sorted; $\sigma_{I} 1.00$ to $2.00 \phi$, poorly sorted; ${ }^{\sigma} I 2.00$ to $4.00 \phi$, very poorly sorted; and $\sigma_{I}$ over $4.00 \phi$, extremely poorly sorted.

Skewness is a measure of the degree of asymmetry of the grain-size distribution. Folk and Ward (1957) proposed the measure inclusive graphic skewness, defined as

$$
S K_{I}=\frac{\phi 16+\phi 84-2 \phi 50}{2(\phi 84-\phi 16)}+\frac{\phi 5+\phi 95-2 \phi 50}{2(\phi 95-\phi 5)}
$$

Symmetrical population curves have $S K_{I}=0$; ones with tails in the fines have positive values to a limit of +1.00 , and ones with tails in the coarse have negative values with a limit of -1.00 . Folk (1968) suggested the following verbal scale for skewness: $S K_{I} 1.00$ to +0.30 , strongly fine skewed; DKI +0.30 to +0.10 , fine skewed; $S K_{I}+0.10$ to -0.10 , near symmetrical; $S K_{I}-0.10$ to 0.30 , coarse skewed; and $S K_{I}-0.30$ to -1.00 , strongly coarse skewed.

Kurtosis or peakedness of the distribution is computed by comparing the spread in the central part of the distribution to the spread in he tails. Folk nd Ward (1957) define graphic kurtosis as

$$
K_{G}=\frac{\phi 95-\phi 5}{2.44(\phi 75-\phi 25)}
$$

Since the distribution of $K_{G}$ values is itself strongly skewed in natural sediments, the kurtosis distribution must be normalized using the formula (Folk and Ward, 1957)

$$
K_{G}^{\prime}=\frac{K_{G}}{1+K_{G}}
$$

They called this value transformed kurtosis and suggested the following verbal classification: $K_{G}^{\prime}$ under 0.40 , very platykurtic; $K_{G}^{\prime} 0.40$ to 0.47 , platykurtic; $K_{G}^{\prime}$ 0.47 to 0.53 , mesokurtic; $K_{G}^{\prime} 0.53$ to 0.60 , leptokurtic; $K_{G}^{\prime} 0.60$ to 0.75 , very leptokurtic; and $K_{G}^{\prime}$ over 0.75 , extremely leptokurtic. For normal probability distributions $K_{G}^{\prime}$ is equal to 0.50 .

Sediment textural classification is that of Shepard (1954), with the sand, silt, and clay boundaries based on the Wentworth (1922) scale. Sand is composed of particles between $2.00 \mathrm{~mm}$ and $62.5 \mu(-1 \phi$ to $+4 \phi)$; silt particles are between $62.5 \mu$ and $3.9 \mu(+4 \phi$ to $+8 \phi)$; and clay is material less than $3.9 \mu(+8 \phi)$.

Replicate analyses on eight standard samples indicate a level of precision of $\pm 3 \%$ for the sand fraction and $\pm 5 \%$ for the clay and silt fractions. Numerous workers (e.g., Maiklem, 1968; Gealy, 1971; and Braithwaite, 1973) have pointed out that skeletal debris (foraminifers, radiolarians, diatoms, etc.) have high specific surface areas so that their settling velocities are slower than their nominal diameter would indicate according to Stokes' formula. Thus, grain-size distributions for biogenous sediments are skewed towards values smaller than their "true" distribution. Grain-size parameters for 

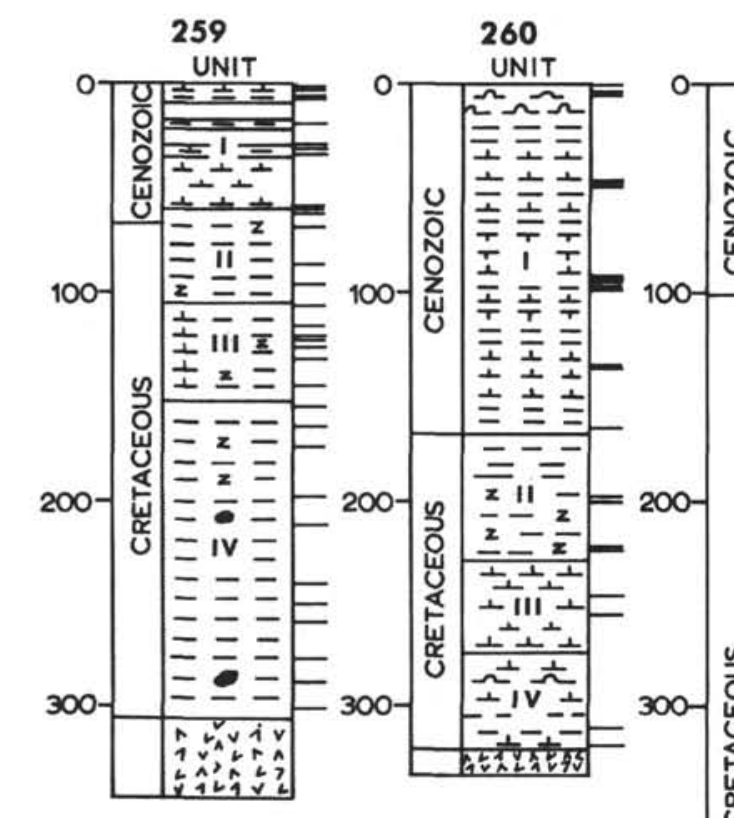

261

262

UNIT

UNIT

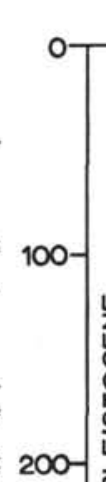

263

ニテニ

=-

-

ニニニ

ㅍェ

国

ニニニ元

프프

ニニ

$---$

-

-

ㅍ--

no

岂 $\overline{\text { IV }}=30$

(1)

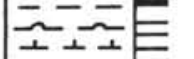

들

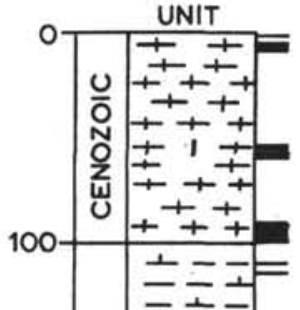

กัュュ

$\approx=$

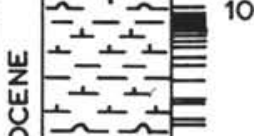

工立屋

ㅍ--

$--$

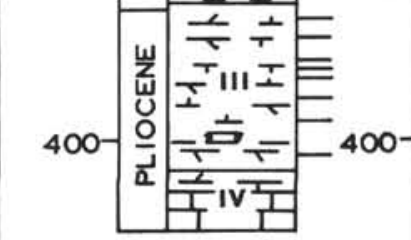

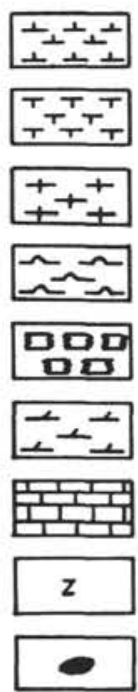

NANNO OOZE

FORAM DOZE

NANNO FORAM OOZE

RAD OOZE

MICARB OOZE

DOLOMITE

LIMESTONE

ZEOLITE

MANGANESE NODULE
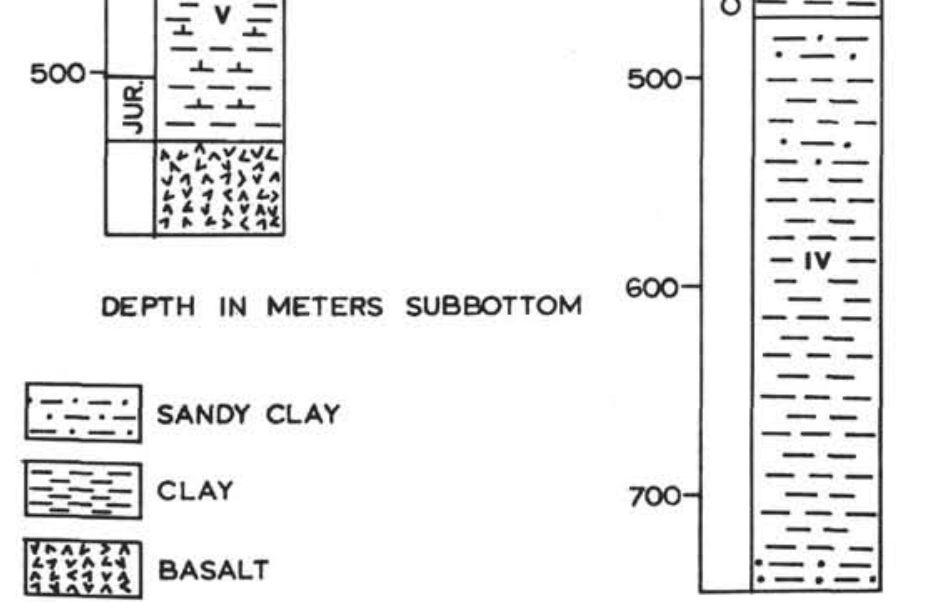

Figure 2. Generalized stratigraphic sections of Leg 27 drill sites with sample locations indicated by horizontal lines.

sediments with abundant radiolarians and diatoms are also smaller than "true" values since their opaline skeletons have densities of $2.2 \mathrm{~g} / \mathrm{cc}$, whereas Stokes' Law assumes density to be $2.67 \mathrm{~g} / \mathrm{cc}$ for all particles.

\section{RESULTS}

Triangular diagrams showing sand-silt-clay percentages for different stratigraphic units at each Leg 17 site are shown in Figure 3. Table 1 lists Folk and Ward (1957) statistical parameters for all samples. Percentages of sand-silt-clay and Shepard's (1954) textural terms for individual samples are tabulated in Table 2 . Table 3 summarizes arithmetic means and standard deviations of statistical parameters for different lithologic units at each site. Details concerning stratigraphic subdivision and lithologic features are discussed in Part I (Site Reports) of this volume. 

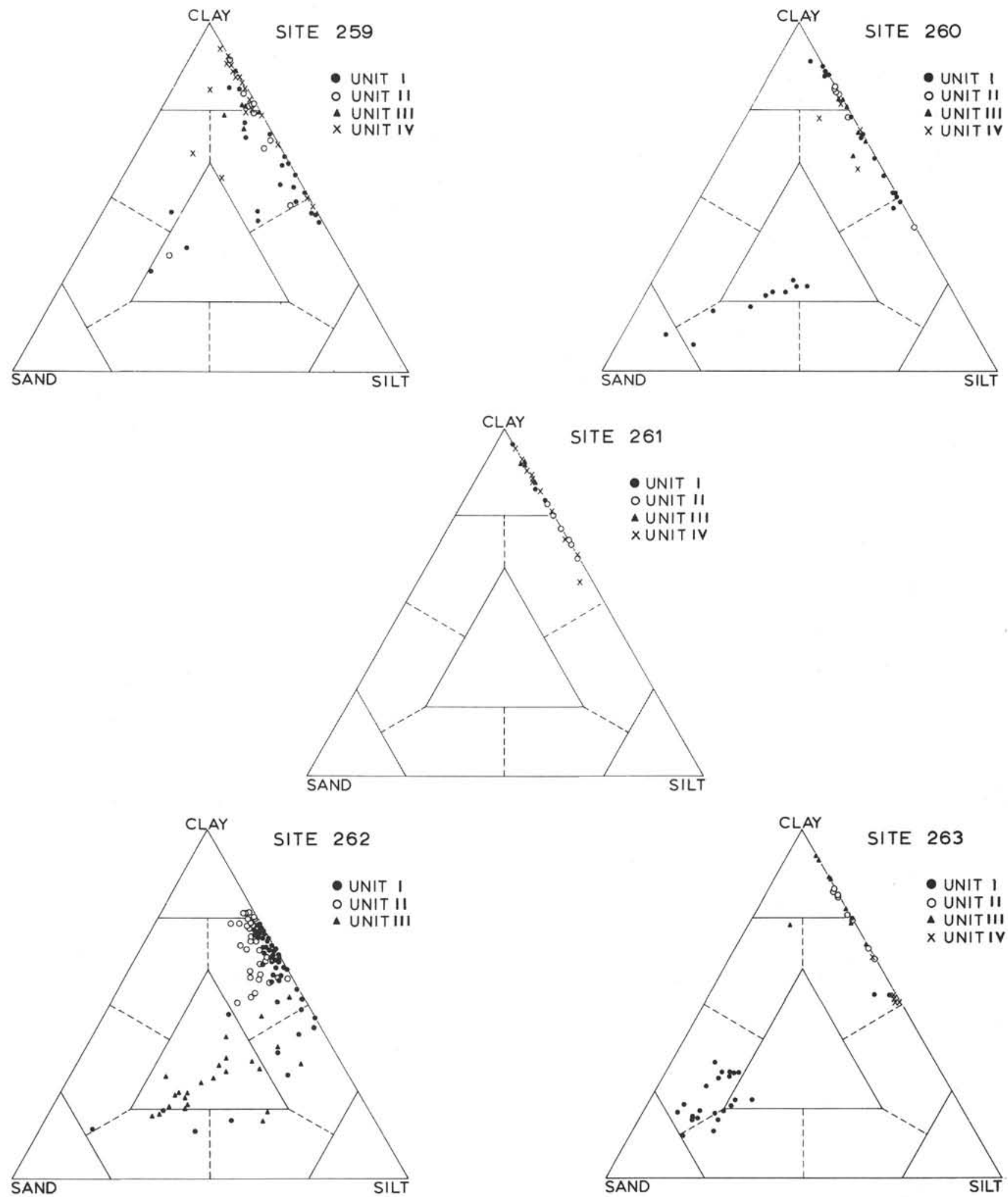

Figure 3. Triangular diagrams showing sand-silt-clay percentages of samples from stratigraphic units at each Leg 27 drilling site. Fields outlined on each diagram are those of Shepard (1954) 
TABLE 1

Folk's Statistical Parameters

\begin{tabular}{|c|c|c|c|c|c|c|}
\hline $\begin{array}{c}\text { Sample } \\
\text { (Interval in } \mathrm{cm} \text { ) }\end{array}$ & Depth $(\mathrm{m})$ & $\phi 5$ & $M_{z}(\phi)$ & $\sigma_{I}(\phi)$ & $S K_{I}$ & $K_{G}^{\prime}$ \\
\hline $259-1-1,122-124$ & 1.2 & 1.11 & 5.02 & 3.77 & 0.51 & 0.40 \\
\hline $259-1-2,90-92$ & 2.41 & 3.04 & 8.37 & 2.87 & -0.15 & 0.58 \\
\hline $259-1-2,93-95$ & 2.42 & 1.67 & 6.95 & 4.11 & 0.15 & 0.39 \\
\hline $259-1-6,74-76$ & 8.25 & 4.58 & 8.94 & 2.61 & 0.11 & 0.48 \\
\hline $259-1, \mathrm{CC}$ & 9.2 & 4.02 & 7.90 & 3.32 & 0.14 & 0.49 \\
\hline $259-3-2,84-86$ & 19.85 & 4.53 & 8.56 & 2.25 & 0.01 & 0.52 \\
\hline $259-4-1,85-87$ & 27.86 & 0.56 & 6.13 & 4.19 & -0.07 & 0.38 \\
\hline $259-4-3,74-76$ & 30.75 & 1.08 & 7.74 & 3.76 & -0.02 & 0.56 \\
\hline $259-4-4,74-76$ & 32.25 & 1.05 & 7.33 & 3.68 & -0.09 & 0.51 \\
\hline $259-7-3,74-76$ & 59.25 & 3.65 & 8.64 & 2.52 & 0.03 & 0.57 \\
\hline $259-7-4,94-95$ & 60.95 & -1.45 & 5.31 & 5.74 & 0.16 & 0.33 \\
\hline $259-7-5,127-129$ & 62.78 & 5.70 & 9.95 & 2.50 & 0.11 & 0.39 \\
\hline $259-8-3,74-76$ & 68.75 & 4.51 & 9.32 & 2.92 & 0.21 & 0.39 \\
\hline $259-10-3,74-76$ & 87.75 & 3.67 & 10.87 & 3.01 & -0.45 & 0.45 \\
\hline $259-11-3,74-76$ & 97.25 & 6.53 & 10.34 & 2.37 & -0.19 & 0.42 \\
\hline $259-12-3,74-76$ & 106.75 & 5.56 & 10.31 & 2.53 & -0.23 & 0.45 \\
\hline $259-13-3,74-76$ & 116.25 & 4.66 & 10.35 & 2.63 & -0.26 & 0.48 \\
\hline $259-13-6,56-58$ & 120.57 & 5.15 & 9.96 & 2.79 & -0.27 & 0.47 \\
\hline $259-13-6,130-132$ & 121.31 & 5.06 & 10.25 & 2.58 & -0.21 & 0.52 \\
\hline $259-14-3,74-76$ & 125.75 & 5.21 & 10.28 & 2.57 & -0.22 & 0.46 \\
\hline $259-14-6,66-68$ & 130.17 & 3.17 & 9.65 & 3.13 & -0.14 & 0.47 \\
\hline $259-14-6,126-128$ & 130.77 & 2.69 & 9.91 & 3.19 & -0.36 & 0.48 \\
\hline $259-16-3,74-76$ & 144.75 & 6.53 & 9.98 & 2.35 & 0.15 & 0.38 \\
\hline $259-17-3,74-76$ & 154.25 & 5.74 & 11.20 & 2.44 & -0.34 & 0.45 \\
\hline $259-18-3,74-76$ & 163.75 & 5.74 & 10.35 & 2.47 & -0.21 & 0.46 \\
\hline $259-19-3,74-76$ & 173.25 & 4.69 & 9.63 & 2.87 & -0.04 & 0.47 \\
\hline $259-22-1,89-91$ & 198.90 & 4.23 & 9.68 & 2.93 & -0.08 & 0.44 \\
\hline $259-23-3,74-76$ & 211.25 & 5.05 & 11.26 & 2.54 & -0.36 & 0.53 \\
\hline $259-26-3,64-66$ & 239.65 & 6.66 & 10.65 & 2.51 & -0.10 & 0.48 \\
\hline $259-27-3,74-76$ & 249.25 & 5.24 & 11.27 & 2.50 & -0.36 & 0.53 \\
\hline $259-28-3,74-76$ & 258.75 & 4.62 & 10.03 & 2.64 & 0.02 & 0.48 \\
\hline $259-30-3,74-76$ & 277.75 & 4.64 & 10.94 & 2.59 & -0.10 & 0.55 \\
\hline $259-31-3,74-76$ & 287.25 & 1.51 & 10.33 & 3.13 & -0.35 & 0.56 \\
\hline $259-33-1,84-86$ & 303.35 & 4.67 & 8.29 & 2.48 & -0.04 & 0.45 \\
\hline $260-1-1,9-11$ & 0.10 & 4.24 & 9.76 & 3.52 & -0.34 & 0.36 \\
\hline $260-1-3,74-76$ & 3.75 & 4.24 & 10.23 & 3.41 & -0.52 & 0.39 \\
\hline $260-1-3,76-78$ & 3.77 & 4.19 & 9.34 & 3.23 & -0.17 & 0.39 \\
\hline $260-2-2,61-63$ & 46.12 & 4.67 & 9.97 & 2.65 & 0.03 & 0.42 \\
\hline $260-2-2,139-141$ & 46.90 & 1.18 & 4.59 & 3.72 & 0.74 & 0.49 \\
\hline $260-2-3,38-40$ & 47.39 & 0.06 & 2.64 & 2.73 & 0.60 & 0.56 \\
\hline $260-2-3,48-50$ & 47.49 & -1.41 & 1.16 & 3.65 & 0.61 & 0.59 \\
\hline $260-3-2,90-92$ & 93.91 & 6.53 & 9.63 & 1.39 & -0.01 & 0.93 \\
\hline $260-3-3,74-76$ & 95.25 & 6.66 & 9.31 & 1.20 & -0.38 & 0.93 \\
\hline $260-3-4,83-85$ & 96.84 & 6.56 & 9.60 & 2.34 & 0.49 & 0.42 \\
\hline $260-3-5,10-12$ & 97.61 & 2.14 & 6.49 & 3.92 & 0.45 & 0.51 \\
\hline $260-3-5,34-36$ & 97.85 & 1.72 & 5.81 & 3.58 & 0.36 & 0.46 \\
\hline $260-3-5,74-76$ & 98.25 & 1.74 & 5.83 & 3.43 & 0.32 & 0.49 \\
\hline $260-3-5,104-106$ & 98.55 & 4.59 & 8.28 & 2.89 & 0.29 & 0.54 \\
\hline $260-3-5,139-141$ & 98.90 & 2.56 & 5.42 & 2.97 & 0.54 & 0.48 \\
\hline $260-3-6,49-51$ & 99.50 & 2.14 & 5.59 & 3.35 & 0.49 & 0.47 \\
\hline $260-3-6,79-81$ & 99.80 & 2.09 & 5.91 & 3.60 & 0.51 & 0.47 \\
\hline $260-3-6,108-110$ & 100.09 & 1.53 & 5.37 & 3.83 & 0.64 & 0.46 \\
\hline $260-3-6,123-125$ & 100.24 & 5.18 & 11.56 & 2.28 & -0.26 & 0.62 \\
\hline $260-4-5,40-44$ & 135.92 & 6.50 & 8.90 & 1.73 & 0.36 & 0.46 \\
\hline $260-4-6,6-8$ & 137.07 & 5.14 & 8.64 & 2.48 & 0.47 & 0.53 \\
\hline $260-5-6,80-82$ & 166.31 & 7.51 & 11.25 & 2.18 & -0.28 & 0.54 \\
\hline $260-7-3,74-76$ & 199.75 & 6.54 & 10.92 & 2.56 & -0.38 & 0.42 \\
\hline $260-7-4,4-6$ & 200.55 & 6.58 & 10.91 & 2.56 & -0.37 & 0.42 \\
\hline $260-8-5,105-107$ & 222.06 & 4.58 & 8.43 & 3.20 & 0.33 & 0.42 \\
\hline $260-8-6,133-135$ & 223,84 & 4.74 & 10.28 & 2.64 & -0.25 & 0.43 \\
\hline $260-10-2,55-59$ & 244.64 & 4.57 & 8.67 & 3.32 & 0.03 & 0.48 \\
\hline $260-11-1,90-92$ & 253.91 & 5.61 & 10.29 & 2.48 & -0.19 & 0.45 \\
\hline $260-17-1,114-116$ & 311.15 & 5.54 & 9.99 & 2.53 & 0.07 & 0.41 \\
\hline $260-18-1,117-119$ & 320.68 & -1.25 & 10.23 & 3.52 & -0.39 & 0.61 \\
\hline $261-3-1,105-107$ & 48.56 & 5.14 & 9.29 & 3.02 & -0.09 & 0.46 \\
\hline
\end{tabular}


TABLE 1 - Continued

\begin{tabular}{|c|c|c|c|c|c|c|}
\hline $\begin{array}{c}\text { Sample } \\
\text { (Interval in } \mathrm{cm} \text { ) }\end{array}$ & Depth (m) & $\phi 5$ & $M_{z}(\phi)$ & $\sigma_{I}(\phi)$ & $S K_{I}$ & $K_{G}^{\prime}$ \\
\hline $261-4-2,13-15$ & 96.64 & 6.56 & 9.96 & 2.30 & 0.13 & 0.60 \\
\hline $261-4-2,60-62$ & 97.11 & 4.51 & 8.92 & 3.35 & -0.18 & 0.37 \\
\hline $261-4-2,79-81$ & 97.30 & 5.07 & 9.91 & 2.56 & 0.05 & 0.47 \\
\hline $261-4-2,102-104$ & 97.53 & 5.62 & 9.38 & 2.43 & -0.13 & 0.60 \\
\hline $261-5-1,95-97$ & 162.46 & 5.20 & 10.06 & 2.79 & -0.32 & 0.41 \\
\hline $261-6-3,84-86$ & 174.85 & 7.54 & 10.99 & 2.12 & 0.05 & 0.46 \\
\hline $261-6-5,49-51$ & 177.50 & 7.65 & 11.63 & 1.88 & -0.16 & 0.54 \\
\hline $261-8-3,74-76$ & 193.75 & 8.51 & 11.64 & 1.74 & -0.11 & 0.50 \\
\hline $261-8-6,94-96$ & 198.45 & 6.61 & 11.02 & 2.54 & -0.40 & 0.48 \\
\hline $261-9-3,0-5$ & 202.53 & 6.55 & 9.88 & 2.32 & 0.20 & 0.49 \\
\hline $261-9-3,74-76$ & 203.25 & 6.65 & 11.27 & 2.32 & -0.33 & 0.57 \\
\hline $261-19-2,140-142$ & $30 t$ & 4.57 & 8.45 & 2.97 & 0.03 & 0.43 \\
\hline $261-19-4,134-137$ & 309.85 & 2.04 & 3.09 & 0.52 & -0.18 & 0.43 \\
\hline $262-1-2,129-131$ & 2.80 & 8.51 & 10.23 & 1.75 & 0.53 & 0.41 \\
\hline $262-1-2,131-132$ & 2.82 & 5.02 & 9.58 & 2.80 & -0.02 & 0.47 \\
\hline $262-1-3,73-75$ & 3.74 & 5.12 & 9.59 & 2.79 & 0.00 & 0.46 \\
\hline $262-1-4,99-101$ & 5.50 & 1.55 & 3.63 & 2.32 & 0.03 & 0.69 \\
\hline $262-2-3,74-76$ & 8.75 & 4.59 & 9.27 & 3.10 & -0.11 & 0.43 \\
\hline $262-3-3,94-96$ & 18.45 & 4.66 & 9.24 & 3.12 & -0.12 & 0.42 \\
\hline $262-3-6,95-97$ & 22.96 & 4.71 & 9.60 & 2.85 & -0.07 & 0.48 \\
\hline $262-4-3,136-138$ & 28.37 & 4.70 & 9.5 & 2.86 & -0.02 & 0.47 \\
\hline $262-5-3,74-76$ & 37.25 & 4.71 & 9.67 & 2.87 & -0.08 & 0.46 \\
\hline $262-5-6,43-45$ & 41.44 & 3.62 & 9.59 & 3.04 & -0.09 & 0.45 \\
\hline $262-6-1,102-104$ & 43.53 & 0.70 & 2.79 & 0.94 & -0.51 & 0.55 \\
\hline $262-6-2,140-142$ & 45.91 & 4.72 & 9.98 & 2.91 & -0.32 & 0.38 \\
\hline $262-6-3,74-76$ & 46.75 & 4.09 & 9.31 & 3.19 & -0.14 & 0.44 \\
\hline $262-6-5,99-101$ & 50.00 & 5.16 & 9.62 & 2.78 & -0.03 & 0.46 \\
\hline $262-6-6,44-46$ & 50.95 & 5.06 & 9.62 & 2.83 & -0.04 & 0.46 \\
\hline $262-7-3,104-106$ & 56.55 & 5.11 & 9.94 & 2.79 & -0.29 & 0.46 \\
\hline $262-7-5,52-54$ & 59.03 & 4.61 & 9.96 & 2.88 & -0.29 & 0.43 \\
\hline $262-8-4,88-90$ & 68. & 4.18 & 9.3 & 3.21 & -0.12 & 0.40 \\
\hline $262-8-5,124-126$ & 69.25 & 4.52 & 8.79 & 3.23 & 0.07 & 0.43 \\
\hline $262-8-6,94-96$ & 70.45 & 5.56 & 9.71 & 2.73 & -0.02 & 0.40 \\
\hline $262-9-1,108-110$ & 72.59 & 4.62 & 8.35 & 2.67 & 0.26 & 0.46 \\
\hline $262-9-3,74-76$ & 75.25 & 5.53 & 9.67 & 2.74 & -0.01 & 0.40 \\
\hline $262-10-3,74-76$ & 84.75 & 5.17 & 9.67 & 2.83 & -0.05 & 0.37 \\
\hline $262-11-3,104-106$ & 94.55 & 4.09 & 9.02 & 3.17 & 0.08 & 0.44 \\
\hline $262-11-6,119-121$ & 99.20 & 3.17 & 7.12 & 3.45 & 0.54 & 0.45 \\
\hline $262-12-1,138-140$ & 101.39 & 4.05 & 8.48 & 3.56 & -0.01 & 0.36 \\
\hline $262-12-2,59-61$ & 102.10 & 3.61 . & 8.60 & 3.49 & 0.02 & 0.3 \\
\hline $262-12-2,103-105$ & 102.53 & 3.09 & 7.97 & 3.84 & 0.12 & 0.3 \\
\hline $262-12-3,74-76$ & 103.75 & 2.72 & 6.49 & 3.76 & 0.64 & 0.3 \\
\hline $262-12-4,9-11$ & 104.60 & 0.66 & 4.48 & 3.72 & 0.46 & 0.5 \\
\hline $262-12-4,129-131$ & 105.80 & 2.54 & 5.29 & 2.91 & 0.61 & 0.73 \\
\hline $262-12-5,112-114$ & 107.13 & 3.05 & 6.91 & 3.50 & 0.66 & 0.4 \\
\hline $262-12-6,52-55$ & 108.03 & 4.56 & 8.61 & 3.34 & 0.08 & 0.38 \\
\hline $262-13-3,74-76$ & 113.25 & 5.06 & 9.34 & 2.38 & -0.18 & 0.50 \\
\hline $262-13-4,115-117$ & 115.16 & 4.51 & 8.30 & 3.36 & 0.28 & 0.42 \\
\hline $262-13-6,123-125$ & 118 & 364 & 8.09 & 3.60 & 0.19 & $0.4 C$ \\
\hline $262-14-3,74-76$ & 2.75 & 5.09 & 9.59 & 2.82 & 0.01 & 0.4 \\
\hline $262-14-5,43-45$ & 125.44 & 4.04 & 8.92 & 3.20 & 0.09 & 0.44 \\
\hline $262-15-3,74-76$ & 132.25 & 4.70 & 9.66 & 2.87 & -0.04 & 0.43 \\
\hline $262-16-3,74-76$ & 141.75 & 4.57 & 9.61 & 2.91 & -0.05 & $0.3 s$ \\
\hline $262-16-4,52-54$ & 143.03 & 2.00 & 4.02 & 2.73 & 0.70 & 0.6 \\
\hline $262-17-3,74-76$ & 151.25 & 4.61 & 9.56 & 2.87 & -0.04 & 0.48 \\
\hline $262-17-5,68-70$ & 154.19 & 4.04 & 8.48 & 3.54 & -0.02 & 0.40 \\
\hline $262-19-3,74-76$ & 170.25 & 4.68 & 9.65 & 2.88 & -0.06 & 0.47 \\
\hline $262-19-6,122-124$ & 175.23 & 4.54 & 9.61 & 2.93 & -0.07 & 0.3 \\
\hline $262-20-3,74-76$ & 179.75 & 5.63 & 9.67 & 2.70 & 0.00 & 0.4 \\
\hline $262-21-3,74-76$ & 189.25 & 5.03 & 9.67 & 2.81 & -0.05 & 0.46 \\
\hline $262-22-3,74-76$ & 198.75 & 5.19 & 10.02 & 2.78 & -0.28 & 0.42 \\
\hline $262-23-3,74-76$ & 208.25 & 5.72 & 10.27 & 2.47 & -0.18 & 0.45 \\
\hline $262-25-3,74-76$ & 227.25 & 5.05 & 9.69 & 2.80 & -0.04 & 0.46 \\
\hline $262-26-3,74-76$ & 236.75 & 5.69 & 9.96 & 2.70 & -0.24 & 0.4 \\
\hline $3,74-76$ & 246.25 & 5.20 & 9.72 & 2.77 & -0.04 & 0.4 \\
\hline $262-28-3,44-46$ & 255.45 & 1.73 & 8.25 & 4.04 & -0.12 & 0.42 \\
\hline
\end{tabular}


TABLE 1 - Continued

\begin{tabular}{|c|c|c|c|c|c|c|}
\hline $\begin{array}{c}\text { Sample } \\
\text { (Interval in cm) }\end{array}$ & Depth (m) & $\phi 5$ & $M_{z}(\phi)$ & $\sigma_{I}(\phi)$ & $S k_{I}$ & $K_{G}^{\prime}$ \\
\hline $262-29-3,74-76$ & 265.25 & 2.53 & 9.68 & 3.21 & -0.17 & 0.49 \\
\hline $262-30-3,74-76$ & 274.75 & 2.22 & 9.17 & 3.60 & -0.25 & 0.48 \\
\hline $262-31-4,89-91$ & 285.90 & 1.67 & 8.58 & 3.84 & -0.10 & 0.45 \\
\hline $262-32-3,74-76$ & 293.75 & 4.50 & 9.34 & 3.16 & -0.16 & 0.39 \\
\hline $262-33-3,74-76$ & 303.25 & 4.12 & 10.27 & 3.19 & -0.30 & 0.44 \\
\hline $262-34-3,74-76$ & 312.75 & 4.51 & 9.61 & 2.92 & -0.08 & 0.43 \\
\hline $262-35-3,74-76$ & 322.25 & 5.06 & 9.66 & 2.82 & 0.04 & 0.42 \\
\hline $262-35-5,39-40$ & 324.90 & 4.56 & 9.65 & 3.13 & -0.36 & 0.39 \\
\hline $262-37-3,66-70$ & 341.18 & 2.69 & 8.25 & 3.66 & 0.16 & 0.41 \\
\hline $262-38-3,68-72$ & 350.70 & 1.71 & 6.50 & 4.00 & 0.42 & 0.44 \\
\hline $262-39-4,49-51$ & 361.50 & 2.23 & 6.61 & 3.81 & 0.49 & 0.44 \\
\hline $262-39-6,49-51$ & 364.50 & 2.11 & 6.63 & 3.83 & 0.48 & 0.44 \\
\hline $262-40-3,77-80$ & 369.78 & 3.12 & 8.99 & 3.64 & -0.25 & 0.38 \\
\hline $262-41-4,139-141$ & 381.40 & 1.64 & 5.79 & 3.71 & 0.58 & 0.48 \\
\hline $262-42-5,113-116$ & 392.15 & 1.62 & 5.80 & 3.72 & 0.55 & 0.49 \\
\hline $262-44-3,74-76$ & 407.75 & 1.14 & 5.73 & 3.89 & 0.42 & 0.39 \\
\hline $263-1-1,145-147$ & 1.46 & 3.65 & 8.63 & 3.46 & 0.00 & 0.41 \\
\hline $263-1-3,124-126$ & 4.25 & 1.05 & 4.10 & 3.51 & 0.80 & 0.62 \\
\hline $263-1-4,10-12$ & 4.61 & 1.02 & 2.93 & 2.54 & 0.68 & 0.61 \\
\hline $263-1-4,100-102$ & 5.52 & 0.65 & 4.07 & 3.54 & 0.65 & 0.57 \\
\hline $263-1-4,118-120$ & 5.69 & 0.67 & 4.64 & 4.33 & 0.77 & 0.54 \\
\hline $263-1-4,139-141$ & 5.90 & 0.08 & 3.83 & 3.88 & 0.67 & 0.55 \\
\hline $263-2-2,15-17$ & 54.16 & 0.75 & 4.84 & 4.17 & 0.68 & 0.38 \\
\hline $263-2-2,70-72$ & 54.71 & 0.73 & 4.97 & 4.16 & 0.59 & 0.41 \\
\hline $263-2-2,122-124$ & 55.23 & 0.70 & 4.64 & 3.96 & 0.58 & 0.41 \\
\hline $263-2-3,40-42$ & 55.91 & 0.72 & 5.01 & 4.18 & 0.58 & 0.41 \\
\hline $263-2-3,124-126$ & 56.75 & 0.56 & 4.92 & 4.23 & 0.57 & 0.40 \\
\hline $263-2-4,10-12$ & 57.11 & 0.58 & 4.99 & 4.37 & 0.58 & 0.42 \\
\hline $263-2-4,19-20$ & 57.19 & 0.10 & 4.99 & 4.54 & 0.46 & 0.39 \\
\hline $263-3-1,110-112$ & 91.61 & 1.21 & 4.61 & 3.69 & 0.74 & 0.50 \\
\hline $263-3-2,69-70$ & 92.70 & 1.67 & 4.64 & 3.30 & 0.72 & 0.53 \\
\hline $263-3-2,109-111$ & 93.10 & 1.64 & 4.23 & 2.85 & 0.68 & 0.57 \\
\hline $263-3-3,74-76$ & 94.25 & 1.63 & 4.64 & 3.29 & 0.70 & 0.59 \\
\hline $263-3-4,50-52$ & 95.51 & 1.18 & 4.14 & 3.34 & 0.81 & 0.60 \\
\hline $263-3-4,124-126$ & 96.25 & 1.23 & 4.59 & 3.70 & 0.75 & 0.55 \\
\hline $263-3-6,100-102$ & 99.01 & 1.08 & 4.77 & 4.02 & 0.83 & 0.48 \\
\hline $263-3-6,142-144$ & 99.43 & 1.14 & 4.46 & 3.52 & 0.62 & 0.45 \\
\hline $263-4-1,9-11$ & 109.60 & 5.50 & 10.61 & 2.72 & -0.17 & 0.45 \\
\hline $263-4-3,74-76$ & 113.25 & 5.67 & 10.27 & 2.50 & -0.20 & 0.41 \\
\hline $263-6-3,74-76$ & 151.25 & 6.51 & 9.61 & 2.34 & 0.44 & 0.42 \\
\hline $263-9-3,77-79$ & 227.28 & 5.71 & 9.93 & 2.46 & 0.07 & 0.46 \\
\hline
\end{tabular}


TABLE 2

Percentage of Sand, Silt, and Clay

\begin{tabular}{|c|c|c|c|c|c|}
\hline $\begin{array}{c}\text { Sample } \\
\text { (Interval in } \mathrm{cm} \text { ) }\end{array}$ & $\begin{array}{l}\text { Depth } \\
\text { (m) }\end{array}$ & $\begin{array}{c}\text { Sand } \\
(\%)\end{array}$ & $\begin{array}{l}\text { Silt } \\
(\%)\end{array}$ & $\begin{array}{l}\text { Clay } \\
(\%)\end{array}$ & $\begin{array}{l}\text { Shepard's } \\
\text { (1954) Textural } \\
\text { Classification }\end{array}$ \\
\hline $259-1-1,122-124$ & 1.20 & 50.7 & 20.9 & 28.4 & Sand-silt-clay \\
\hline $259-1-2,90-92$ & 2.41 & 7.3 & 25.4 & 67.3 & Silty clay \\
\hline $259-1-2,93-95$ & 2.42 & 37.0 & 17.1 & 45.9 & Sandy clay \\
\hline $259-1-6,74-76$ & 8.25 & 2.1 & 38.7 & 59.2 & Silty clay \\
\hline $259-1, \mathrm{CC}$ & 9.20 & 3.9 & 47.2 & 48.9 & Silty clay \\
\hline $259-3-2,84-86$ & 19.85 & 2.7 & 44.3 & 53.0 & Silty clay \\
\hline $259-4-1,85-87$ & 27.86 & 38.1 & 26.1 & 35.8 & Sand-silt-clay \\
\hline $259-4-3,74-76$ & 30.75 & 15.0 & 38.8 & 46.2 & Silty clay \\
\hline $259-4-4,74-76$ & 32.25 & 16.3 & 40.6 & 43.1 & Silty clay \\
\hline $259-7-3,74-76$ & 59.25 & 5.1 & 41.0 & 53.9 & Silty clay \\
\hline $259-7-4,94-95$ & 60.95 & 43.9 & 22.6 & 33.5 & Sand-silt-clay \\
\hline $259-7-5,127-129$ & 62.78 & 1.3 & 32.4 & 66.3 & Silty clay \\
\hline $259-8-3,74-76$ & 68.75 & 0.1 & 43.3 & 56.7 & Silty clay \\
\hline $259-10-3,74-76$ & 87.75 & 5.4 & 23.2 & 71.4 & Silty clay \\
\hline $259-11-3,75-76$ & 97.25 & 1.1 & 22.4 & 76.5 & Clay \\
\hline $259-12-3,74-76$ & 106.75 & 3.0 & 20.2 & 76.8 & Clay \\
\hline $259-13-3,74-76$ & 116.25 & 4.4 & 14.2 & 81.4 & Clay \\
\hline $259-13-6,56-58$ & 120.57 & 1.6 & 21.5 & 76.9 & Clay \\
\hline $259-13-6,130-132$ & 121.31 & 3.7 & 20.1 & 76.2 & Clay \\
\hline $259-14-3,74-76$ & 125.75 & 3.0 & 21.4 & 75.6 & Clay \\
\hline $259-14-6,66-68$ & 130.17 & 6.7 & 23.3 & 70.0 & Silty clay \\
\hline $259-14-6,126-128$ & 130.77 & 9.9 & 16.2 & 73.9 & Silty clay \\
\hline $259-16-3,74-76$ & 144.75 & 0.5 & 25.2 & 74.3 & Silty clay \\
\hline $259-17-3,74-76$ & 154.25 & 0 & 13.5 & 86.5 & Clay \\
\hline $259-18-3,74-76$ & 163.75 & 0.2 & 18.5 & 81.3 & Clay \\
\hline $259-19-3,74-76$ & 173.25 & 0.2 & 34.2 & 65.6 & Silty clay \\
\hline $259-22-1,89-91$ & 198.90 & 0.1 & 30.9 & 68.9 & Silty clay \\
\hline $259-23-3,74-76$ & 211.25 & 0.4 & 11.7 & 87.9 & Clay \\
\hline $259-26-3,64-66$ & 239.65 & 0.1 & 17.7 & 82.2 & Clay \\
\hline $259-27-3,74-76$ & 249.25 & 0.1 & 9.4 & 90.5 & Clay \\
\hline $259-28-3,74-76$ & 258.75 & 1.2 & 19.5 & 79.3 & Clay \\
\hline $259-30-3,74-76$ & 277.75 & 0.1 & 13.8 & 86.1 & Clay \\
\hline $259-31-3,74-76$ & 287.25 & 9.6 & 9.4 & 81.0 & Clay \\
\hline $259-33-1,84-86$ & 303.35 & 0.3 & 49.5 & 50.2 & Silty clay \\
\hline $260-1-1,9-11$ & 0.10 & 0.7 & 43.0 & 56.3 & Silty clay \\
\hline $260-1-3,74-76$ & 3.75 & 0.8 & 30.6 & 68.6 & Silty clay \\
\hline $260-1-3,76-78$ & 3.77 & 0.4 & 31.7 & 67.9 & Silty clay \\
\hline $260-2-2,61-63$ & 46.12 & 0.1 & 26.8 & 73.1 & Silty clay \\
\hline $260-2-2,139-141$ & 46.90 & 63.3 & 19.4 & 17.3 & Silty sand \\
\hline $260-2-3,38-40$ & 47.39 & 73.1 & 19.1 & 7.8 & Silty sand \\
\hline $260-2-3,48-50$ & 47.49 & 78.7 & 10.8 & 10.5 & Sand \\
\hline $260-3-2,90-92$ & 93.91 & 0 & 12.4 & 87.6 & Clay \\
\hline $260-3-3,74-76$ & 95.25 & 0.1 & 13.8 & 86.1 & Clay \\
\hline $260-3-4,83-85$ & 96.84 & 0 & 38.7 & 61.3 & Silty clay \\
\hline $260-3-5,10-12$ & 97.61 & 36.0 & 39.9 & 24.1 & Sand-silt-clay \\
\hline $260-3-5,34-36$ & 97.85 & 41.2 & 32.2 & 26.6 & Sand-silt-clay \\
\hline $260-3-5,74-76$ & 98.25 & 41.4 & 34.3 & 24.3 & Sand-silt-clay \\
\hline $260-3-5,104-106$ & 98.55 & 2.7 & 50.2 & 47.1 & Clayey silt \\
\hline $260-3-5,139-141$ & 98.90 & 42.2 & 35.3 & 22.5 & Sand-silt-clay \\
\hline $260-3-6,49-51$ & 99.50 & 47.9 & 30.7 & 21.4 & Sand-silt-clay \\
\hline $260-3-6,79-81$ & 99.80 & 45.5 & 31.9 & 22.6 & Sand-silt-clay \\
\hline $260-3-6,108-110$ & 100.09 & 53.1 & 28.6 & 18.3 & Silty sand \\
\hline $260-3-6,123-125$ & 100.24 & 2.8 & 8.2 & 89.0 & Cay \\
\hline $260-4-5,40-44$ & 135.92 & 0.4 & 48.6 & 51.0 & Clay \\
\hline $260-4-6,6-8$ & 137.07 & 0 & 51.1 & 48.9 & Clayey silt \\
\hline $260-5-6,80-82$ & 166.31 & 0.2 & 14.6 & 85.2 & Clay \\
\hline $260-7-3,74-76$ & 199.75 & 0.1 & 21.1 & 78.9 & Clay \\
\hline $260-7-4,4-6$ & 200.55 & 0.5 & 18.4 & 81.1 & Clay \\
\hline $260-8-5,105-107$ & 222.06 & 0 & 58.5 & 41.5 & Clayey silt \\
\hline $260-8-6,133-135$ & 223.84 & 1.5 & 25.6 & 72.9 & Silty clay \\
\hline $260-10-2,55-59$ & 244.64 & 0.3 & 33.9 & 65.8 & Silty clay \\
\hline $260-11-1,90-92$ & 253.91 & 0.3 & 23.8 & 75.9 & Cay \\
\hline $260-17-1,114-116$ & 311.15 & 0 & 30.8 & 69.2 & Silty clay \\
\hline $260-18-1,117-119$ & 320.68 & 8.8 & 18.3 & 72.9 & Silty clay \\
\hline
\end{tabular}


TABLE 2 - Continued

\begin{tabular}{|c|c|c|c|c|c|}
\hline $\begin{array}{c}\text { Sample } \\
\text { (Interval in } \mathrm{cm} \text { ) }\end{array}$ & $\begin{array}{l}\text { Depth } \\
\text { (m) }\end{array}$ & $\begin{array}{l}\text { Sand } \\
(\%)\end{array}$ & $\begin{array}{l}\text { Silt } \\
(\%)\end{array}$ & $\begin{array}{l}\text { Clay } \\
(\%)\end{array}$ & $\begin{array}{c}\text { Shepard's } \\
\text { (1954) Textural } \\
\text { Classification }\end{array}$ \\
\hline $261-3-1,105-107$ & 48.56 & 0 & 32.1 & 67.9 & Silty clay \\
\hline $261-4-2,13-15$ & 96.64 & 0.3 & 21.5 & 78.2 & Clay \\
\hline $261-4-2,60-62$ & 97.11 & 0.5 & 36.6 & 62.9 & Silty clay \\
\hline $261-4-2,79-81$ & 97.30 & 0.4 & 28.5 & 71.1 & Silty clay \\
\hline $261-4-2,102-104$ & 97.53 & 0 & 24.8 & 75.2 & Clay \\
\hline $261-5-1,95-97$ & 162.46 & 0.9 & 31.0 & 68.1 & Silty clay \\
\hline $261-6-3,84-86$ & 174.85 & 0.2 & 9.5 & 90.3 & Clay \\
\hline $261-6-5,49-51$ & 177.50 & 0.2 & 5.2 & 94.6 & Clay \\
\hline $261-8-3,74-76$ & 193.75 & 0.2 & 4.4 & 95.4 & Clay \\
\hline $261-8-6,94-96$ & 198.45 & 0 & 16.2 & 83.8 & Clay \\
\hline $261-9-3,0-5$ & 202.53 & 0 & 23.6 & 76.4 & Clay \\
\hline $261-9-3,74-76$ & 203.25 & 0.2 & 14.1 & 85.7 & Clay \\
\hline $261-19-2,140-142$ & 306.91 & 3.3 & 41.0 & 55.7 & Silty clay \\
\hline $261-19-4,134-137$ & 309.85 & 99.9 & 0 & 0.1 & Sand \\
\hline $262-1-2,129-131$ & 2.80 & 0 & 3.9 & 96.1 & Clay \\
\hline $262-1-2,131-132$ & 2.82 & 0 & 39.6 & 60.4 & Silty clay \\
\hline $262-1-3,73-75$ & 3.74 & 0.1 & 30.7 & 69.2 & Silty clay \\
\hline $262-1-4,99-101$ & 5.50 & 47.0 & 39.4 & 13.6 & Silty sand \\
\hline $262-2-3,74-76$ & 8.75 & 2.5 & 34.8 & 62.7 & Silty clay \\
\hline $262-3-3,94-96$ & 18.45 & 2.9 & 36.4 & 60.7 & Silty clay \\
\hline $262-3-6,95-97$ & 22.96 & 0.8 & 31.6 & 67.6 & Silty clay \\
\hline $262-4-3,136-138$ & 28.37 & 1.6 & 34.3 & 64.1 & Silty clay \\
\hline $262-5-3,74-76$ & 37.25 & 1.7 & 33.2 & 65.1 & Silty clay \\
\hline $262-5-6,43-45$ & 41.44 & 5.9 & 32.5 & 61.6 & Silty clay \\
\hline $262-6-2,140-142$ & 45.91 & 3.1 & 26.9 & 70.0 & Silty clay \\
\hline $262-6-3,74-76$ & 46.75 & 3.0 & 30.5 & 66.5 & Silty clay \\
\hline $262-6-5,99-101$ & 50.00 & 1.8 & 29.1 & 69.1 & Silty clay \\
\hline $262-6-6,44-46$ & 50.95 & 3.3 & 27.9 & 68.8 & Silty clay \\
\hline $262-7-3,104-106$ & 56.55 & 0.8 & 33.6 & 65.6 & Silty clay \\
\hline $262-7-5,52-54$ & 59.03 & 2.3 & 31.0 & 66.7 & Silty clay \\
\hline $262-8-4,88-90$ & 68.89 & 2.8 & 27.8 & 69.4 & Silty clay \\
\hline $262-8-5,124-126$ & 69.25 & 4.5 & 37.0 & 58.5 & Silty clay \\
\hline $262-8-6,94-96$ & 70.45 & 1.2 & 28.3 & 70.5 & Silty clay \\
\hline $262-9-1,108-110$ & 72.59 & 2.3 & 48.9 & 48.8 & Clayey silt \\
\hline $262-9-3,74-76$ & 75.25 & 2.1 & 28.4 & 69.5 & Silty clay \\
\hline $262-10-3,74-76$ & 84.75 & 1.6 & 33.0 & 65.4 & Silty clay \\
\hline $262-11-3,104-106$ & 94.55 & 3.2 & 30.8 & 66.0 & Silty clay \\
\hline $262-11-6,119-121$ & 99.20 & 14.6 & 49.2 & 36.2 & Clayey silt \\
\hline $262-12-1,138-140$ & 101.39 & 0.5 & 45.1 & 54.4 & Silty clay \\
\hline $262-12-2,59-61$ & 102.10 & 7.3 & 41.9 & 50.8 & Silty clay \\
\hline $262-12-2,103-105$ & 102.53 & 21.7 & 31.2 & 47.1 & Sand-silt-clay \\
\hline $262-12-3,74-76$ & 103.75 & 38.2 & 34.0 & 27.8 & Sand-silt-clay \\
\hline $262-12-4,9-11$ & 104.60 & 52.0 & 28.2 & 19.8 & Silty sand \\
\hline $262-12-4,129-131$ & 105.80 & 36.2 & 47.2 & 16.6 & Sandy silt \\
\hline $262-12-5,112-114$ & 107.13 & 15.2 & 54.8 & 30.0 & Clayey silt \\
\hline $262-12-6,52-55$ & 108.03 & 0.6 & 47.5 & 51.9 & Silty clay \\
\hline $262-13-3,74-76$ & 113.25 & 1.8 & 27.0 & 71.2 & Silty clay \\
\hline $262-13-4,115-117$ & 115.16 & 2.8 & 53.5 & 43.7 & Clayey silt \\
\hline $262-13-6,123-125$ & 118.24 & 6.7 & 51.9 & 41.4 & Clayey silt \\
\hline $262-14-3,74-76$ & 122.75 & 1.6 & 31.4 & 67.0 & Silty clay \\
\hline $262-14-5,43-45$ & 125.44 & 3.9 & 39.1 & 57.0 & Silty clay \\
\hline $262-15-3,74-76$ & 132.25 & 0.7 & 29.1 & 70.2 & Silty clay \\
\hline $262-16-3,74-76$ & 141.75 & 1.5 & 27.6 & 70.9 & Silty clay \\
\hline $262-16-4,52-54$ & 143.03 & 72.2 & 13.6 & 14.2 & Clayey sand \\
\hline $262-17-3,74-76$ & 151.25 & 2.3 & 33.9 & 63.8 & Silty clay \\
\hline $262-17-5,68-70$ & 154.19 & 2.5 & 39.4 & 58.1 & Silty clay \\
\hline $262-19-3,74-76$ & 170.25 & 1.9 & 27.7 & 70.4 & Silty clay \\
\hline $262-19-6,122-124$ & 175.23 & 3.5 & 31.5 & 65.0 & Silty clay \\
\hline $262-20-3,74-76$ & 179.75 & 1.6 & 28.3 & 70.1 & Silty clay \\
\hline $262-21-3,74-76$ & 189.25 & 2.8 & 25.6 & 71.6 & Silty clay \\
\hline $262-22-3,74-76$ & 198.75 & 0.8 & 24.4 & 74.8 & Silty clay \\
\hline $262-23-3,74-76$ & 208.25 & 2.2 & 21.1 & 76.7 & Clay \\
\hline $262-25-3,74-76$ & 227.25 & 3.9 & 24.2 & 71.9 & Silty clay \\
\hline $262-26-3,74-76$ & 236.75 & 2.7 & 22.5 & 74.8 & Silty clay \\
\hline $262-27-3,74-76$ & 246.25 & 2.8 & 20.7 & 76.5 & Clay \\
\hline $262-28-3,44-46$ & 255.45 & 17.5 & 31.9 & 50.6 & Silty clay \\
\hline
\end{tabular}


TABLE 2 - Continued

\begin{tabular}{|c|c|c|c|c|c|}
\hline $\begin{array}{c}\text { Sample } \\
\text { (Interval in } \mathrm{cm} \text { ) }\end{array}$ & $\begin{array}{l}\text { Depth } \\
\text { (m) }\end{array}$ & $\begin{array}{c}\text { Sand } \\
(\%)\end{array}$ & $\begin{array}{l}\text { Silt } \\
(\%)\end{array}$ & $\begin{array}{l}\text { Clay } \\
(\%)\end{array}$ & $\begin{array}{c}\text { Shepard's } \\
\text { (1954) Textural } \\
\text { Classification }\end{array}$ \\
\hline $262-29-3,74-76$ & 265.25 & 7.8 & 18.4 & 73.8 & Silty clay \\
\hline $262-30-3,74-76$ & 274.75 & 8.2 & 25.7 & 66.1 & Silty clay \\
\hline $262-31-4,89-91$ & 285.90 & 11.7 & 34.8 & 53.5 & Silty clay \\
\hline $262-32-3,74-76$ & 293.75 & 5.0 & 32.6 & 62.4 & Silty clay \\
\hline $262-33-3,74-76$ & 303.25 & 4.0 & 23.6 & 72.4 & Silty clay \\
\hline $262-34-3,74-76$ & 312.75 & 4.1 & 30.1 & 65.8 & Silty clay \\
\hline $262-35-3,74-76$ & 322.25 & 3.7 & 27.9 & 68.4 & Silty clay \\
\hline $262-35-5,39-40$ & 324.90 & 3.1 & 31.7 & 65.2 & Silty clay \\
\hline $262-37-3,66-70$ & 341.18 & 13.5 & 40.1 & 46.4 & Silty clay \\
\hline $262-38-3,68-72$ & 350.70 & 30.2 & 39.0 & 30.8 & Sand-silt-clay \\
\hline $262-39-4,49-51$ & 361.50 & 31.4 & 36.0 & 32.6 & Sand-silt-clay \\
\hline $262-39-6,49-51$ & 364.50 & 28.9 & 36.5 & 34.6 & Sand-silt-clay \\
\hline $262-40-3,77-80$ & 369.78 & 10.6 & 32.0 & 57.4 & Silty clay \\
\hline $262-41-4,139-141$ & 381.40 & 45.6 & 30.3 & 24.4 & Sand-silt-clay \\
\hline $262-42-5,113-116$ & 392.15 & 43.5 & 32.1 & 24.4 & Sand-silt-clay \\
\hline $262-44-3,74-76$ & 407.75 & 46.4 & 24.2 & 29.4 & Sand-silt-clay \\
\hline $263-1-1,145-147$ & 1.46 & 5.8 & 41.6 & 52.6 & Silty clay \\
\hline $263-1-3,124-126$ & 4.25 & 72.4 & 9.0 & 18.6 & Cayey sand \\
\hline $263-1-4,10-12$ & 4.61 & 74.6 & 13.3 & 12.1 & Silty sand \\
\hline $263-1-4,100-102$ & 5.52 & 68.3 & 14.6 & 17.1 & Clayey sand \\
\hline $263-1-4,118-120$ & 5.69 & 69.6 & 13.3 & 17.1 & Clayey sand \\
\hline $263-1-4,139-141$ & 5.90 & 69.3 & 13.2 & 17.5 & Clayey sand \\
\hline $263-2-2,15-17$ & 54.16 & 61.2 & 12.4 & 26.4 & Clayey sand \\
\hline $263-2-2,70-72$ & 54.71 & 55.1 & 14.4 & 30.5 & Clayey sand \\
\hline $263-2-2,122-124$ & 55.23 & 57.1 & 14.5 & 28.4 & Clayey sand \\
\hline $263-2-3,40-42$ & 55.91 & 53.2 & 16.4 & 30.4 & Clayey sand \\
\hline $263-2-3,124-126$ & 56.75 & 54.0 & 16.8 & 29.2 & Clayey sand \\
\hline $263-2-4,10-12$ & 57.11 & 52.8 & 17.1 & 30.1 & Clayey sand \\
\hline $263-2-4,19-20$ & 57.19 & 55.6 & 14.7 & 33.7 & Clayey sand \\
\hline $263-3-1,110-112$ & 91.61 & 66.4 & 13.7 & 19.9 & Clayey sand \\
\hline $263-3-2,69-70$ & 92.70 & 63.1 & 18.3 & 18.6 & Clayey sand \\
\hline $263-3-2,109-111$ & 93.10 & 66.0 & 20.2 & 13.8 & Silty sand \\
\hline $263-3-3,74-76$ & 94.25 & 60.5 & 20.1 & 19.4 & Silty sand \\
\hline $263-3-4,50-52$ & 95.51 & 69.9 & 13.2 & 16.9 & Clayey sand \\
\hline $263-3-4,124-126$ & 96.25 & 63.2 & 20.1 & 16.7 & Silty sand \\
\hline $263-3-6,100-102$ & 99.01 & 69.8 & 9.1 & 21.1 & Clayey sand \\
\hline $263-3-6,142-144$ & 99.43 & 57.7 & 21.3 & 21.0 & Sand-silt-clay \\
\hline $263-4-1,9-11$ & 109.60 & 0.8 & 23.2 & 76.0 & Clay \\
\hline $263-4-3,74-76$ & 113.25 & 0.1 & 25.7 & 74.2 & Silty clay \\
\hline $263-6-3,74-76$ & 151.25 & 0.1 & 33.9 & 66.0 & Silty clay \\
\hline $263-9-3,77-79$ & 227.28 & 0.1 & 25.4 & 74.5 & Silty clay \\
\hline
\end{tabular}


TABLE 3

Arithmetic Mean and Standard Deviation of Folk Parameters for Lithologic Units

\begin{tabular}{|c|c|c|c|c|c|c|}
\hline Unit & Samples & $\phi 5$ & $M_{z}(\phi)$ & $\sigma_{I}(\phi)$ & $S K_{I}$ & $K_{G}^{\prime}$ \\
\hline \multicolumn{7}{|c|}{ Site 259} \\
\hline I & 10 & $2.52 \pm 1.59$ & $7.56 \pm 1.24$ & $3.31 \pm 0.70$ & $0.06 \pm 0.19$ & $0.49 \pm 0.08$ \\
\hline II & 5 & $3.79 \pm 3.12$ & $9.15 \pm 2.22$ & $3.31 \pm 1.39$ & $0.03 \pm 0.28$ & $0.39 \pm 0.04$ \\
\hline III & 9 & $4.86 \pm 1.22$ & $10.21 \pm 0.44$ & $2.69 \pm 0.29$ & $-0.21 \pm 0.15$ & $0.46 \pm 0.04$ \\
\hline IV & 10 & $4.70 \pm 1.32$ & $10.24 \pm 0.90$ & $2.67 \pm 0.23$ & $-0.16 \pm 0.15$ & $0.50 \pm 0.04$ \\
\hline \multicolumn{7}{|c|}{ Site 260} \\
\hline I & 22 & $3.62 \pm 2.34$ & $7.51 \pm 2.78$ & $2.93 \pm 0.80$ & $0.22 \pm 0.39$ & $0.52 \pm 0.15$ \\
\hline II & 4 & $5.61 \pm 1.09$ & $10.14 \pm 1.18$ & $2.74 \pm 0.31$ & $-0.17 \pm 0.34$ & $0.42 \pm 0.01$ \\
\hline III & 2 & $5.09 \pm 0.73$ & $9.48 \pm 1.14$ & $2.90 \pm 0.59$ & $-0.08 \pm 0.16$ & $0.46 \pm 0.02$ \\
\hline IV & 2 & $2.14 \pm 4.80$ & $10.11 \pm 0.17$ & $3.02 \pm 0.70$ & $-0.16 \pm 0.32$ & $0.51 \pm 0.14$ \\
\hline \multicolumn{7}{|c|}{ Site 261} \\
\hline II & 5 & $5.37 \pm 0.76$ & $9.49 \pm 0.44$ & $2.73 \pm 0.44$ & $-0.04 \pm 0.13$ & $0.50 \pm 0.01$ \\
\hline III & 7 & $6.96 \pm 1.06$ & $10.93 \pm 0.70$ & $2.24 \pm 0.37$ & $-0.15 \pm 0.22$ & $0.49 \pm 0.05$ \\
\hline IV & 2 & $3.31 \pm 1.79$ & $5.77 \pm 3.79$ & $1.74 \pm 1.73$ & $-0.07 \pm 0.15$ & $0.43 \pm 0.00$ \\
\hline \multicolumn{7}{|c|}{ Site 262} \\
\hline I & 47 & $4.25 \pm 1.32$ & $8.59 \pm 1.79$ & $2.97 \pm 0.49$ & $0.05 \pm 0.26$ & $0.45 \pm 0.08$ \\
\hline II & 14 & $4.12 \pm 1.45$ & $9.56 \pm 0.58$ & $3.10 \pm 0.45$ & $-0.17 \pm 0.10$ & $0.44 \pm 0.03$ \\
\hline III & 8 & $2.03 \pm 0.64$ & $6.79 \pm 1.21$ & $3.78 \pm 0.12$ & $0.36 \pm 0.27$ & $0.43 \pm 0.04$ \\
\hline \multicolumn{7}{|c|}{ Site 263} \\
\hline I & 21 & $1.05 \pm 0.74$ & $4.70 \pm 1.03$ & $3.74 \pm 0.52$ & $0.64 \pm 0.17$ & $0.49 \pm 0.08$ \\
\hline II & 3 & $5.89 \pm 0.54$ & $10.16 \pm 0.51$ & $2.52 \pm 0.19$ & $0.02 \pm 0.36$ & $0.46 \pm 0.02$ \\
\hline
\end{tabular}

\section{Site 259}

Thirty-four samples were analyzed from Site 259. Vertical variations in grain-size parameters for this site are plotted in Figure 4.

Unit I is a clay-rich nanno ooze and zeolite clay. It is chiefly a silty clay, although a few sand-silt-clay layers (foram-bearing nanno oozes) occur (Figure 3). The coarsest $5 \%$ of the distribution $(\phi 5)$, analogous to Carozzi's (1958) clasticity, averages $2.52 \phi(174 \mu)$ and shows no vertical trend. Mean size decreases from $5.02 \phi$ $(30.8 \mu)$ at the top of the unit to $8.94 \phi(2.0 \mu)$ near the base and averages $7.56 \phi(5.3 \mu)$. The sediments are poorly sorted (sample average $=\bar{x}=3.31 \phi$ ) and mesokurtic $(\bar{x}=0.49)$. Skewness is near symmetrical $(\bar{x}=$ $0.06)$. Sorting becomes better near the base and skewness shows an erratic decrease from 0.51 at the top to 0.01 at the base. Transformed kurtosis shows no vertical trend.

Unit II is a dark, yellow-brown zeolitic silty clay (Figure 3 ). Phi 5 averaged $72 \mu(3.79 \phi)$ and average mean size is $1.8 \mu(9.15 \phi)$. The unit is poorly sorted $(\bar{x}=3.31 \phi)$ and very platykurtic $(\bar{x}=0.39)$; skewness is near symmetrical $(\bar{x}=0.03)$. Mean size and phi 5 decrease downward, and sorting becomes slightly better near the base of the unit. Sediments are fine skewed at the top and become coarse skewed near the base. Transformed kurtosis shows a slight increase near the base of the unit.

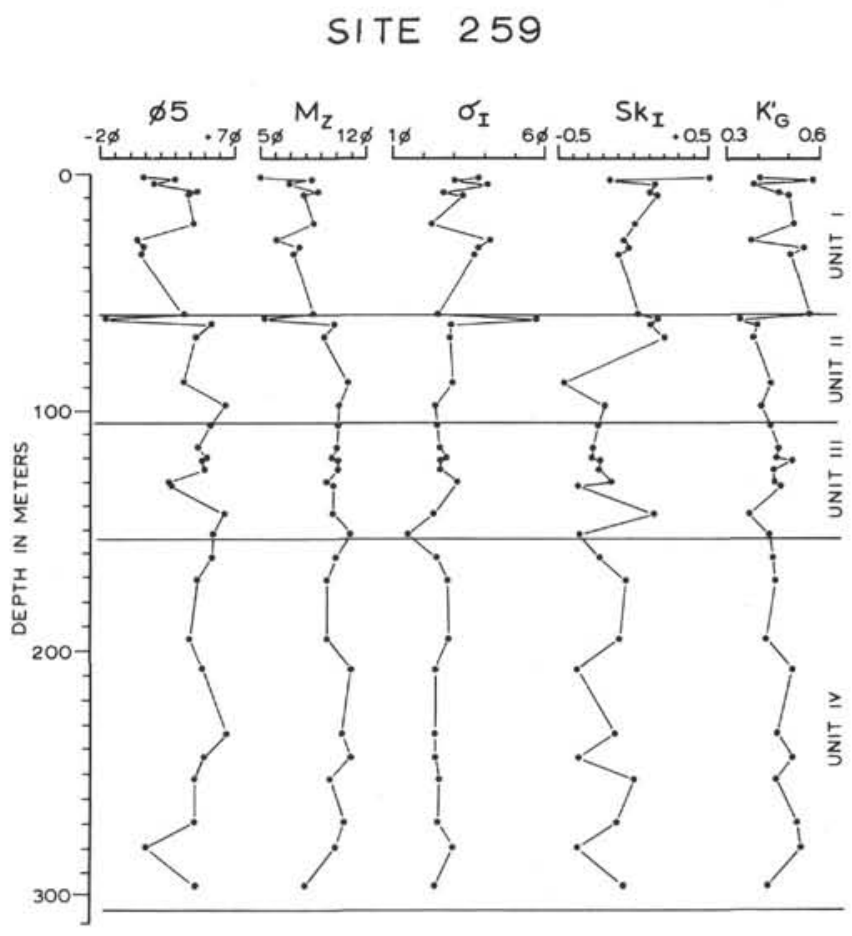

Figure 4. Vertical variation in grain-size parameters of Mesozoic-Cenozoic sediments at DSDP, Site 259. 
Light brown zeolite-rich nanno clay and clayey nanno ooze comprise Unit III (Figure 3). Phi 5 averages $34.4 \mu$ $(4.86 \phi)$ and becomes slightly finer near the base. Mean size averages $10.21 \phi(0.84 \mu)$ and shows no trend. The sediments are better sorted $(x=2.69 \phi)$ than those of Units I or II. They are coarse skewed $(x=-0.21)$ and platykurtic $(x=0.46)$. Sorting becomes better near the base; otherwise, there are no trends.

Unit IV is a greenish-gray zeolite-bearing clay (Figure 3) that displays little vertical variability in grain-size parameters (Figure 4). The coarsest $5 \%$ of the distribution averages $4.7 \phi(38.5 \mu)$, and average mean size is $10.24 \phi(0.83 \mu)$. Average values for sorting, skewness, and kurtosis are nearly identical to those for Unit III and are listed in Table 3.

\section{Site 260}

Thirty samples were analyzed from this site. Because of intermittent coring, a vertical variation diagram of grain-size parameters could not be constructed.

Unit I is a nanno ooze with lesser amounts of brown clay, rad ooze, and graded detrital foram ooze. Texturally, this unit is extremely variable (Figure 3). The brown clays and nanno oozes are chiefly silty clay and clay, whereas the detrital foram oozes are sand-silt-clay, silty sand, and sand. Phi 5 averages $81 \mu(3.63 \phi)$ and mean size is $5.5 \mu(7.51 \phi)$. Most sediments are very poorly sorted $(\bar{x}=2.93 \phi)$, although a few of the clays attain slightly better sorting values (Table 1). Skewness averages 0.22 (fine skewed) and transformed kurtosis 0.52 (mesokurtic). Because of the wide variety of lithologic types included in this unit, average values for statistical parameters display large standard deviations (Table 3).

Figure 5 illustrates vertical variation in sand-silt-clay percentage, mean size, and sorting of one of the carbonate turbidite sequences in Unit I. The sequence is sharply bounded on both sides by zeolite-bearing nanno-rich clays. Except for the break at 98 meters, there is a systematic increase in percent sand (foraminifers) along with a concomitant decrease in silt and clay percentages toward the base of the unit. Because of the high percentage of silt and clay in this unit, mean size lies within the silt range. Like percent sand, mean size increases toward the base of the unit. Sorting values are poorest at the top and base of the unit and best near the middle.

Unit II is a brown zeolitic clay and clay. Average mean values for this unit are: phi $5,5.61 \phi(20.5 \mu)$; mean size, $10.14 \phi(0.89 \mu)$; sorting, $2.74 \phi$ (very poorly sorted); skewness, -0.17 (coarse skewed); and transformed kurtosis, 0.42 (platykurtic).

Unit III is chiefly a nanno ooze with minor brown clay, and Unit IV consists of nanno ooze, rad ooze, and zeolitic clay. Grain-size data (Figure 3, Tables 1, 2, and 3 ) are based on only two samples from each unit and cannot be considered representative for the entire units.

\section{Site 261}

Because of poor core recovery and induration of the sediments, only 14 samples were analyzed. Additional sand-silt-clay data from DSDP grain-size analyses

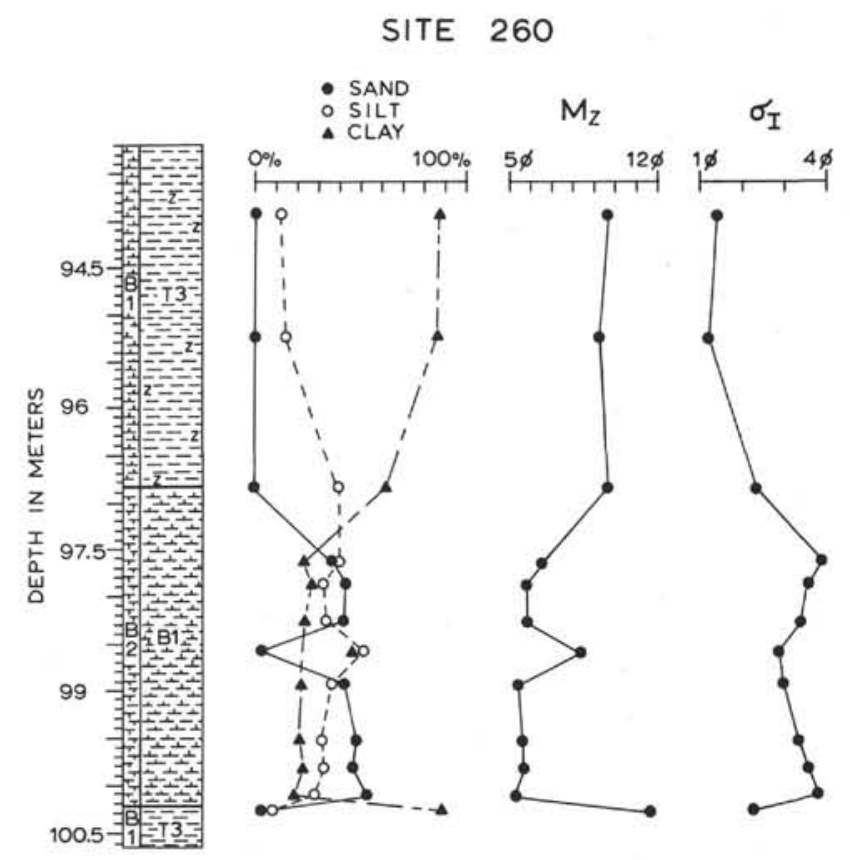

Figure 5. Vertical variation in sand-silt-clay percentage, mean size $\left(M_{\mathrm{Z}}\right)$, and sorting ( $\left.\sigma_{\mathrm{I}}\right)$ of Middle Miocene (?) fine carbonate turbidite sequence at Site 260. The sequence is bounded on both sides by zeolite bearing nanno rich clays.

(Bode, Chapter 22, this volume) were utilized in constructing the textural plot shown in Figure 3. The most conspicuous textural feature of the sediments at this site is that all fall in the clay and silty clay fields and that there is virtually no sand present. Only Units II and III were sampled in sufficient detail to warrant discussion.

Unit II consists of greenish-gray nanno ooze and nanno-rich clay. Average mean values for statistical parameters are: phi $5,5.37 \phi(24 \mu)$; mean size, $9.49 \phi$ $(1.39 \mu)$; sorting, $2.73 \phi$ (very poorly sorted); skewness, -0.04 (near symmetrical); and transformed kurtosis, 0.50 (mesokurtic).

Unit III is a gray, zeolite-bearing clay with the following average values for statistical parameters: phi $5,8 \mu$ (6.96 $\phi)$; mean size, $0.51 \mu(10.93 \phi)$; sorting, $2.24 \phi$ (very poorly sorted); skewness, -0.15 (coarse skewed); and transformed kurtosis, 0.49 (mesokurtic).

\section{Site 262}

Because of continuous coring, good recovery, and the unconsolidated nature of the sediments, 69 samples were analyzed from the upper three units at this site (Figure $2)$. Vertical variations in grain-size parameters are plotted in Figure 6.

Unit I is a grayish-olive rad and clay-rich nanno ooze with subordinate detrital foram sands. Most samples are silty clays (Figure 3 ). The coarsest $5 \%$ averages $4.25 \phi$ $(52 \mu)$, and mean size is $8.59 \phi(2.59 \mu)$. The sediments are very poorly sorted $(\bar{x}=2.97 \phi)$, near symmetrical $(\bar{x}=$ $0.05^{\prime}$, and platykurtic $(\bar{x}=0.45)$. Generally, there are no systematic vertical variations in grain-size parameters in this unit. There are, however, sharp peaks between $0-10$, 
40-50, 100-110, and 140-150 meters. These peaks result from the occurrence of detrital foram sands at these depths. The foram sands are turbidites that are generally coarser grained and better sorted than the associated nanno oozes of Unit I. These sands also tend to be strongly fine skewed and very leptokurtic (Figure 6).

Figure 7 illustrates one of the carbonate turbidite sequences composed of foram sand-silt-clay, silty sand, and sandy silt that occurs between 101 and 108.5 meters. This may represent a multiple turbidite sequence because (1) there is no sharp break between the detrital foram sand and overlying foram sandy silt at 104.5 meters, and (2) the detrital foram sand does not display a systematic upward fining of mean size or an upward decrease in percent sand. The detrital foram sands display slightly better sorting values than the associated nanno oozes, and they are more fine skewed.

Unit II is a grayish-olive micarb and clay-rich nanno ooze. The great bulk of Unit II samples are silty clays (Figure 3 ). Phi 5 averages $4.12 \phi(57.5 \mu)$, although values between $1 \phi$ and $2 \phi$ are common from 250 to 285 meters. Mean size averages $9.56 \phi(1.32 \mu)$ and shows a slight coarsening towards the base of the unit. Sorting averages $3.10 \phi$ (very poorly sorted) and becomes poorer toward the base. Average skewness is -0.17 (coarse skewed); values become positive (i.e., near symmetrical and fine skewed) near the base. Transformed kurtosis averages 0.44 (platykurtic) and shows no trend.

Unit III, a grayish-olive nanno-rich foram ooze, has the following average values: phi $5,2.03 \phi(245 \mu)$; mean size, $6.79 \phi(9 \mu)$; sorting $3.78 \phi$ (very poorly sorted); skewness, 0.36 (strongly fine skewed); and transformed

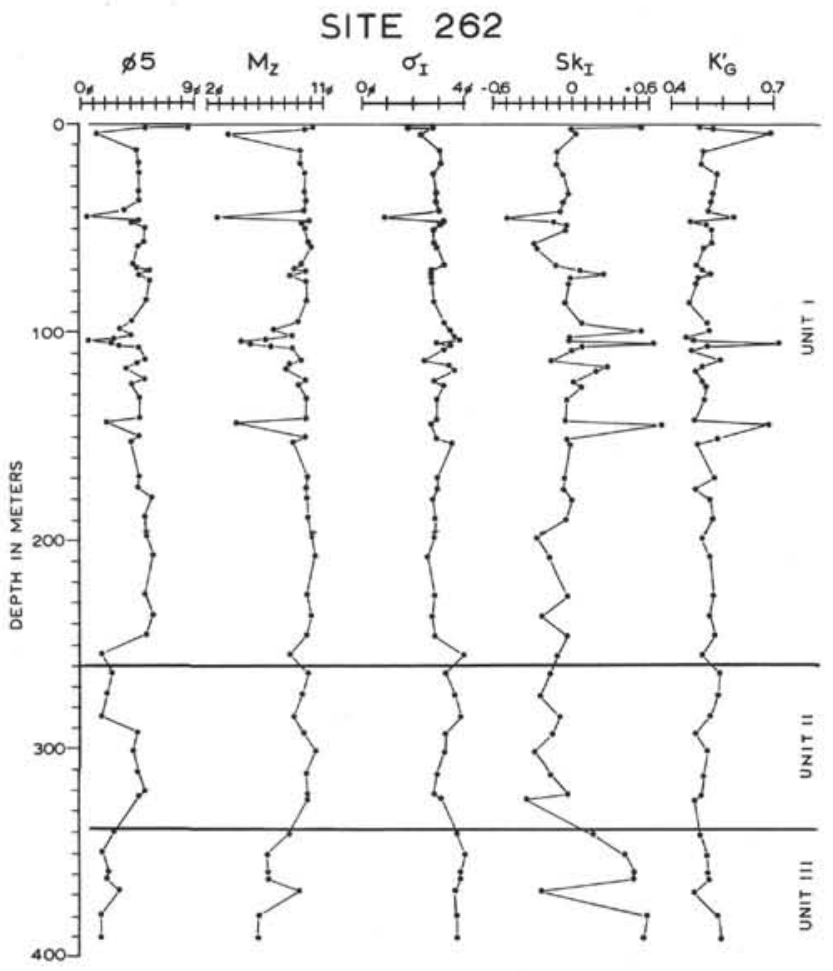

Figure 6. Vertical variation in grain-size parameters of Pliocene-Pleistocene sediments at DSDP, Site 262.

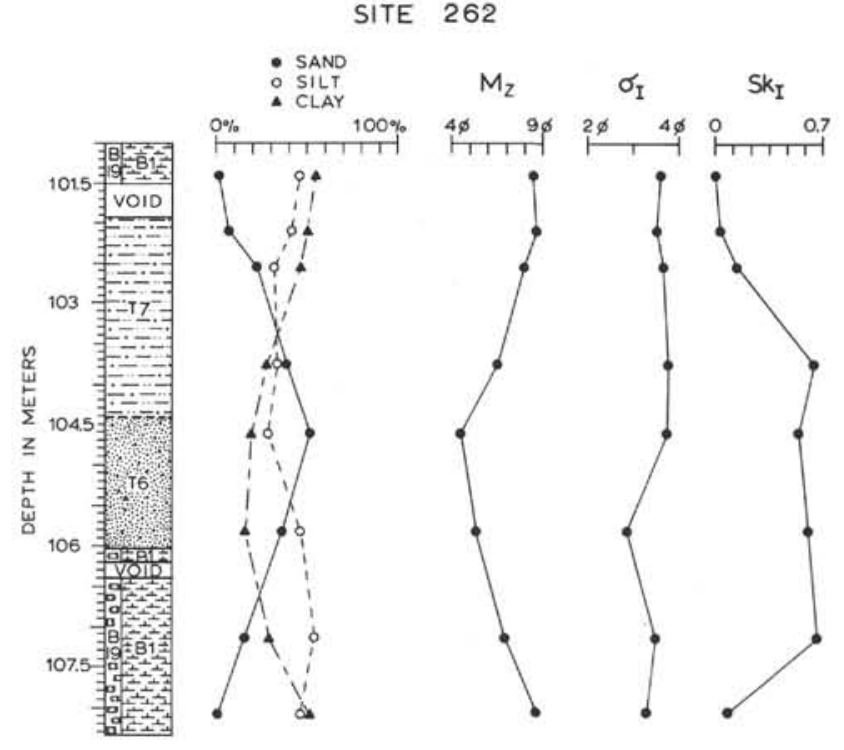

Figure 7. Vertical variation in sand-silt-clay percentage, mean size $\left(M_{\mathrm{Z}}\right)$, and sorting $\left(\sigma_{I}\right)$ of Pleistocene-Holocene turbidite sequence at Site 262. The sequence is bounded on both sides by micarb rich nanno ooze. T7 is a foram sand-silt-clay and T6 is foram silty sand and sandy silt.

kurtosis, 0.43 (platykurtic). Unit III samples display wide textural variability (Figure 3 ) because of varying proportions of foraminifers. Most samples, however, fall in the sand-silt-clay field of Shepard (1954). Phi 5 and mean size become coarser near the base, whereas sorting shows no trend. Skewness increases towards higher positive values (i.e., becomes strongly fine skewed) near the base. Transformed kurtosis increases slightly in a downward direction.

\section{Site $\mathbf{2 6 3}$}

Twenty-five samples were analyzed from this site and most of these (21) are from Unit I. Grain-size analyses were not performed on Units III and IV because of their induration. Sand-silt-clay ratios shown in Figure 3 for Units III IV are from Bode (this volume). Sediments in Units II, III, and IV are dominantly clays and silty clays. Because of the high foram content of Unit I sediments, they lie chiefly in the clayey sand and silty sand textural fields (Figure 3).

Unit I is dominantly a detrital foram nanno ooze with minor nanno ooze. Phi 5 averages $473 \mu(1.05 \phi)$ and mean size is $38.5 \mu(4.70 \phi)$. The coarse size of both parameters is attributable to the large percentage of foraminifers that occur in this unit. The sediments are very poorly sorted $(\bar{x}=3.74)$, strongly fine skewed $(\bar{x}=$ $0.64)$, and mesokurtic $(\bar{x}=0.49)$.

Unit II is a greenish-gray to olive black clay, nannobearing clay, and clayey nanno ooze. Average mean values for this unit are: phi $5,5.89 \phi(16.8 \mu)$; mean size, $10.16 \phi(0.87 \mu)$; sorting, $2.52 \phi$ (very poorly sorted); skewness, 0.02 (near symmetrical); and transformed kurtosis, 0.46 (platykurtic). 


\section{DISCUSSION}

Cook (Chapter 20, this volume) has demonstrated that there are marked geochemical differences in composition of Mesozoic and Cenozoic sediments cored at Leg 27 sites. This observation is supported by the carbonate percentage data of Bode (Chapter 21, this volume) and by the X-ray mineralogical data of Cook, Zemmels, and Matti (this volume).

These data show that the basic difference between the two is in the percentage of carbonate. Mesozoic sediments are composed chiefly of quartz and clay minerals, with varying amounts of zeolites and minor carbonate. In contrast, Cenozoic sediments are dominantly composed of carbonate (mostly calcite), with subordinate quartz and minor clay minerals and zeolites.

Grain-size results from this study also indicate differences between Mesozoic and Cenozoic sediments (Table 4). Generally, Cenozoic sediments are coarser grained, more poorly sorted, and finer skewed than Mesozoic ones. Transformed kurtosis values are similar for both.

TABLE 4

Average Grain-Size Parameters by Age

\begin{tabular}{ccc}
\hline & $\begin{array}{c}\text { Mesozoic } \\
\text { Sediments } \\
\text { (44 Samples) }\end{array}$ & $\begin{array}{c}\text { Cenozoic } \\
\text { Sediments } \\
(128 \text { Samples) }\end{array}$ \\
\hline$M_{z}$ & $10.07 \phi \pm 1.35 \phi$ & $7.67 \phi \pm 2.26 \phi$ \\
$\sigma_{I}$ & $2.58 \phi \pm 0.48 \phi$ & $3.19 \phi \pm 0.68 \phi$ \\
$S K_{I}$ & $-0.14 \quad \pm 0.21$ & $+0.18 \pm 0.34$ \\
$K_{G}^{\prime}$ & $0.47 \pm 0.05$ & $0.47 \pm 0.09$ \\
\hline
\end{tabular}

It should be pointed out that part of these differences may be due to biased sampling techniques, which included: (1) preferential sampling of Cenozoic sediments over Mesozoic ones by a factor of almost three to one, and (2) preferential sampling of coarse-grained Cenozoic sediments over fine-grained ones. However, since the grain-size data are in close agreement with the geochemical and mineralogical evidence, these dif- ferences are believed to be real and not due to sample bias.

Average mean size of Mesozoic sediments lies in the clay size class $(0.92 \mu)$ while that of Cenozoic sediments is in the very fine silt class $(4.91 \mu)$. Mean size of both is a function of sediment composition (Table 5). Mesozoic sediments are predominantly zeolite clays, quartz and cristobalite clays, and nanno clays; hence, their mean size falls in the clay range. Cenozoic sediments, on the other hand, are calcareous oozes of two basic types: (1) foram oozes whose mean size falls in the medium and coarse silt range ( $4.89 \phi$ to $5.86 \phi)$; and (2) nanno oozes whose mean size lies in the clay range $(9.14 \phi$ to $9.52 \phi)$ (Table 5). The mean size of $7.67 \phi$ (fine silt) for Cenozoic sediments results from averaging the coarse and medium silt-size foram oozes with clay-size nanno oozes.

Mesozoic sediments have an average sorting value $\left(\sigma_{\mathrm{I}}\right)$ of $2.58 \phi$ while Cenozoic sediments average $3.19 \phi$. Both are very poorly sorted according to the terminology of Folk and Ward (1957). As Folk (1968) has pointed out, sorting is strongly dependent on mean grain size. A scatter plot of mean size versus sorting for Mesozoic and Cenozoic sediments reveals the following: (1) the best-sorted sediments have mean diameters of about $3 \phi$; (2) the worst-sorted sediments have mean sizes between $5.5 \phi$ and $8 \phi$; and (3) below $8 \phi$ sorting becomes better with increasing mean size, so that best-sorted sediments in the clay range have mean diameters between $10 \phi$ and $11 \phi$. Since Mesozoic sediments consist dominantly of one size population (i.e., clay), they display the best sorting values. Cenozoic sediments, in contrast, are mixtures of two different size populations (sand and coarse silt and clay); hence, they have poorer sorting values.

Mesozoic sediments are coarse skewed $(\bar{x}=-0.14)$, i.e., they have an excess of coarse material. Cenozoic sediments have an excess of fine material and are fine skewed $(\bar{x}=+0.18)$. Folk and Ward (1957) have shown that skewness values which depart from normality (greater than +0.1 or less than -0.1 ) result from subequal mixing of two normal size populations. Mesozoic sediments consist of a dominant fine clay population along with a subordinate coarse population (sand- and silt-size grains of zeolites, quartz, radiolarians, etc.).

TABLE 5

Average Grain-Size Parameters for Major Lithologic Types

\begin{tabular}{lrrrrr}
\hline Lithology & Samples & $M_{z}(\phi)$ & $\sigma_{I}(\phi)$ & \multicolumn{1}{c}{$S K_{I}$} & $K_{G}^{\prime}$ \\
\hline Foram nanno ooze & 37 & 5.86 & 3.51 & +0.48 & 0.48 \\
Micarb nanno ooze & 8 & 8.69 & 3.31 & +0.06 & 0.41 \\
Rad nanno ooze & 17 & 9.14 & 2.99 & +0.03 & 0.44 \\
Clay nanno ooze & 36 & 9.47 & 2.85 & -0.13 & 0.45 \\
Nanno ooze & 9 & 9.52 & 2.91 & -0.04 & 0.46 \\
Nanno foram ooze & 8 & 5.51 & 3.66 & +0.42 & 0.46 \\
Foram ooze & 8 & 4.89 & 2.91 & +0.39 & 0.56 \\
Rad ooze & 3 & 9.77 & 3.39 & -0.34 & 0.38 \\
Zeolite clay & 20 & 9.52 & 2.89 & -0.11 & 0.45 \\
Quartz and & & & & & \\
$\quad$ cristobalite clay & 12 & 10.07 & 2.67 & -0.13 & 0.49 \\
Nanno clay & 10 & 10.25 & 2.15 & +0.01 & 0.56 \\
\hline
\end{tabular}


This slight excess of coarse material produces the negative skewness. Cenozoic sediments have a dominant coarse population (sand and coarse silt), which is mixed with a subordinate fine clay population (chiefly clay minerals and broken coccoliths). They have more fine material than a normal population should have, and thus, are positively skewed.

Transformed kurtosis averages 0.47 (mesokurtic) for both Mesozoic and Cenozoic sediments. As noted previously, normal probability distributions have transformed kurtosis values equal to 0.50 . The slight departure of Mesozoic and Cenozoic sediments from normality is probably a consequence of subequal mixing of two different size populations (Folk and Ward, 1957). In Mesozoic sediments this results from mixing of a dominant fine population (clay) with a subordinate coarse one (sand and coarse silt). The departure from normality of Cenozoic sediments results from mixing a dominant coarse population (sand and coarse silt) with a subordinate clay one.

\section{CONCLUSIONS}

1. Folk and Ward grain-size parameters can be used to distinguish unconsolidated stratigraphic units at Leg 27 sites.

2. Almost all samples analyzed have mean sizes in the silt and clay range and are very poorly sorted. Skewness and kurtosis values exhibit greater variability.

3. Grain-size results from this study indicate marked variability between Mesozoic and Cenozoic sediments. Generally, Cenozoic sediments are coarser grained, more poorly sorted, and finer skewed than Mesozoic ones. Kurtosis values are similar for both.

4. Sorting is strongly dependent on mean size. Sediments with mean sizes between 5.5 and $8 \phi$ have the poorest sorting values. Below $8 \phi$ sorting becomes better with increasing mean size, so that best-sorted sediments are in the clay range with mean diameters between $10 \phi$ and $11 \phi$.

5. Values for skewness and kurtosis show slight departures from those predicted by the normal probability distribution. These departures most likely result from subequal mixing of fine and coarse sediment populations.

\section{ACKNOWLEDGMENTS}

The writers thank the Program in Marine Sciences Research of the University of North Carolina at Wilmington for providing space, equipment, and computer time necessary for completion of this project. The Program also provided summer research salary for Thayer during 1973.

\section{REFERENCES}

Barth, T. F. W., Correns, C. W., and Eskola, P., 1939. Die Entstehung der Gesteine: Berlin (Springer).

Beall, A. O., Laury, R., Dickinson, K., and Pusey, W. C., III, 1973. Sedimentology, In Worzel, J. L., Bryant, W. et al., Initial Reports of the Deep Sea Drilling Project, Volume 10: Washington (U.S. Government Printing Office), p. 699.

Blatt, H., Middleton, G., and Murray, R., 1972. Origin of sedimentary rocks: Englewood Cliffs, N. J. (Prentice-Hall, Inc.).
Braithwaite, C. J. R., 1973. Settling behaviour related to sieve analysis of skeletal sands: Sedimentology, v. 20, p. 251.

Boyce, R. E., 1972. Leg 11 grain size analysis. In Hollister, C. D., Ewing, J. I., et al., Initial Reports of the Deep Sea Drilling Project, Volume 11: Washington (U.S. Government Printing Office), p. 1047.

Carozzi, A. V., 1958. Micro-mechanisms of sedimentation in the epicontinental environment: J. Sediment. Petrol., v. 28, p. 133.

Correns, C. W., 1955. Pelagic sediments of the North Atlantic Ocean. In Trask, P. D. (Ed.), Recent marine sediments: Tulsa (Society of Economic Paleontologists and Mineralogists), p. 373.

Folk, R. L., 1968. Petrology of sedimentary rocks. Austin, Texas (Hemphill's Book Store).

Folk, R. L. and Ward, W. C., 1957. Brazos River bar, a study in the significance of grain size parameters: J. Sediment. Petrol., v. 27, p. 3.

Galehouse, J. S., 1971. Sedimentation analysis. In Carver, R. E. (Ed.), Procedures in sedimentary petrology. New York (Wiley-Interscience), p. 69.

Gealy, E. L., 1971. Grain size of sediments from the western equatorial Pacific: Leg 7, Glomar Challenger. In Winterer, E. L. et al., Initial Reports of the Deep Sea Drilling Project, Volume 7: Washington (U.S. Government Printing Office), p. 1027.

Griffiths, J. C., 1967. Scientific method in analysis of sediments: New York (McGraw-Hill).

Ingram, R. L., 1971. Sieve analysis. In Carver, R. E. (Ed.), Procedures in sedimentary petrology: New York (WileyInterscience), p. 49.

Inman, D. L., 1962. Measures for describing the size distribution of sediments: J. Sediment. Petrol., v. 22, p. 125.

Irani, R. R. and Callis, C. F., 1963. Particle size: Measurement, interpretation, and application: New York (Wiley).

Krumbein, W. C. and Pettijohn, F. J., 1938. Manual of sedimentary petrography: New York (Appleton-CenturyCrofts, Inc.).

Kuenen, Ph. H., 1950. Marine geology: New York (Wiley).

Kuenen, Ph. H. and Neeb, G. A., 1943. Bottom samples: The Snellius Expedition: v. 5, part 3, Leyden (Brill).

Kukal, Zdenek, 1971. Geology of recent sediments: London (Academic Press).

Lisitzin, A. P., 1972. Sedimentation in the world ocean: Soc. Econ. Paleontol. Mineral. Spec. Publ. 17.

Lisitzin, A. P., Serova, V. V., Zverinskaya, I. B., Lukashin, V., Gorbunova, Z. N., Gordeev, V. V., Zhurensko, V. V., Pchelintsev, A. M., Belaev, Jw. I., Popov, N. I., Shishkina, O. V., Morozov, N. M., Jouse, A. P., Kozlova, O. G., Mukhina, V. V., Khodkevich, Yu. N., and Plyusnina, I. I., 1971. Geochemical, mineralogical, and paleontological studies. In Fischer, A. G. et al., Initial Reports of the Deep Sea Drilling Project, Volume 6: Washington (U.S. Government Printing Office), p. 829.

Maiklem, W. R., 1968. Some hydraulic properties of bioclastic carbonate grains: Sedimentology, v. 10, p. 101.

Müller, G., 1967. Sedimentary petrology, Part I: Methods in sedimentary petrology: New York (Hafner Publishing Co.).

Revelle, R. R., 1944. Marine bottom samples collected in the Pacific Ocean by the Carnegie on its seventh cruise: Carnegie Inst. Publ. 556, Part 1.

Shepard, F. P., 1954. Nomenclature based on sand-silt-clay ratios: J. Sediment. Petrol., v. 24, p. 151.

Shukri, N. M. and Higazy, R. A., 1944. Mechanical analyses of some bottom deposits of the northern Red Sea: J. Sediment. Petrol., v. 14, p. 43.

Sverdrup, H. U., Johnson, M. W., and Fleming, R. H., 1942. The oceans: Englewood Cliffs, N. J. (Prentice-Hall, Inc.). 
Trask, P. D., 1932. Origin and environment of source sediments of petroleum: Houston (Gulf Publishing Co.).

van Andel, Tj. H., 1964. Recent marine sediments of Gulf of California. In van Andel, Tj. H. and Shor, G. G., Jr. (Eds.), Marine geology of the Gulf of California: Am. Assoc. Petrol. Geol. Mem. 3, p. 216. van Andel, T. H. and Veevers, J. J., 1967. Morphology and sediments of the Timor Sea: Bur. Min. Res. Australia Bull. 83.

Wentworth, C. K., 1922. A scale of grade and class terms for clastic sediments: J. Geol., v. 30, p. 377. 